\title{
Elliptic curves of rank two and generalised Kato classes
}

Henri Darmon ${ }^{1 *}$ and Victor Rotger ${ }^{2}$

\author{
${ }^{*}$ Correspondence: \\ darmon@math.mcgill.ca \\ 'Department of Mathematics \\ and Statistics, McGill University, \\ 805 Sherbrooke St. West, \\ Montreal, Canada \\ Full list of author information is \\ available at the end of the article
}

In memory of Robert Coleman.

\begin{abstract}
Heegner points play an outstanding role in the study of the Birch and Swinnerton-Dyer conjecture, providing canonical Mordell-Weil generators whose heights encode first derivatives of the associated Hasse-Weil L-series. Yet the fruitful connection between Heegner points and $L$-series also accounts for their main limitation, namely that they are torsion in (analytic) rank $>1$. This partly expository article discusses the generalised Kato classes introduced in Bertolini et al. (J Algebr Geom 24:569-604, 2015) and Darmon and Rotger (J AMS 2016), stressing their analogy with Heegner points but explaining why they are expected to give non-trivial, canonical elements of the idoneous Selmer group in settings where the classical L-function (of Hasse-Weil-Artin type) that governs their behaviour has a double zero at the centre. The generalised Kato class denoted $\kappa(f, g, h)$ is associated to a triple $(f, g, h)$ consisting of an eigenform $f$ of weight two and classical $p$-stabilised eigenforms $g$ and $h$ of weight one, corresponding to odd two-dimensional Artin representations $V_{g}$ and $V_{h}$ of $G a l(H / \mathbb{Q})$ with $p$-adic coefficients for a suitable number field $H$. This class is germane to the Birch and Swinnerton-Dyer conjecture over $H$ for the modular abelian variety $E$ over $\mathbb{Q}$ attached to $f$. One of the main results of Bertolini et al. (2015) and Darmon and Rotger (J AMS 2016) is that $\kappa(f, g, h)$ lies in the pro-p Selmer group of $E$ over $H$ precisely when $L\left(E, V_{g h}, 1\right)=0$, where $L\left(E, V_{g h}, s\right)$ is the $L$-function of $E$ twisted by $V_{g h}:=V_{g} \otimes V_{h}$. In the setting of interest, parity considerations imply that $L\left(E, V_{g h}, s\right)$ vanishes to even order at $s=1$, and the Selmer class $\kappa(f, g, h)$ is expected to be trivial when $\operatorname{ord}_{s=1} L\left(E, V_{g h}, s\right)>2$. The main new contribution of this article is a conjecture expressing $\kappa(f, g, h)$ as a canonical point in $\left(E(H) \otimes V_{g h}\right)^{G} \mathbb{Q}$ when $\operatorname{ord}_{s=1} L\left(E, V_{g h}, s\right)=2$. This conjecture strengthens and refines the main conjecture of Darmon et al. (Forum Math Pi 3:e8, 2015) and supplies a framework for understanding the results of Darmon et al. (2015), Bertolini et al. (2015) and Darmon and Rotger (J AMS 2016).
\end{abstract}

Mathematics Subject Classification: 11G18, 14G35

\section{Contents}

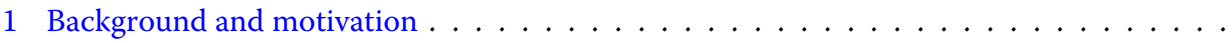

2 Hida families and periods for weight one forms $\ldots \ldots \ldots \ldots \ldots \ldots$

3 Generalised Kato classes . . . . . . . . . . . . . . . . . . . . . . . . . . . . . . .

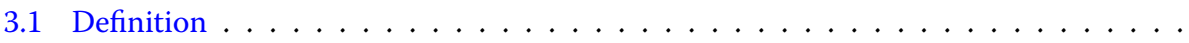

3.2 Basic properties . . . . . . . . . . . . . . . . . . .

(c) 2016 The Author(s). This article is distributed under the terms of the Creative Commons Attribution 4.0 International License (http://creativecommons.org/licenses/by/4.0/), which permits unrestricted use, distribution, and reproduction in any medium, provided you give appropriate credit to the original author(s) and the source, provide a link to the Creative Commons license, and indicate if changes were made. 
3.3 Enhanced regulators $\ldots \ldots \ldots \ldots \ldots \ldots \ldots$

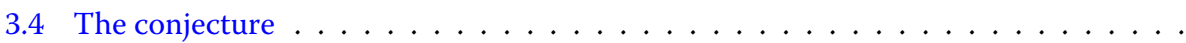

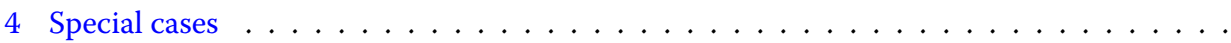

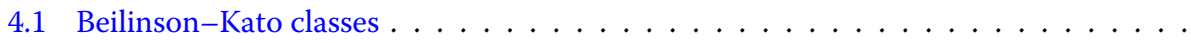

4.2 Beilinson-Flach classes . . . . . . . . . . . . . . . .

4.3 Complex multiplication classes and Heegner points . . . . . . . . . . . .

4.4 Real multiplication classes and Stark-Heegner points . . . . . . . . . . . . . .

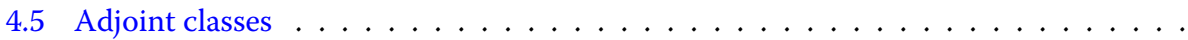

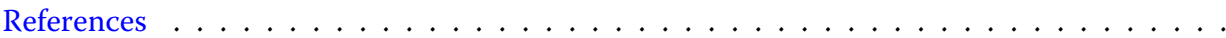

\section{Background and motivation}

The theme of modularity of $p$-adic Galois representations has occupied centre stage in number theory for the last several decades, and Robert Coleman has been a major figure in many of its key developments, notably through the theory of Coleman families of $p$-adic modular forms and of the Coleman-Mazur eigencurve parameterising these families and their associated Galois representations. By way of background and motivation, this section explains how much of the progress achieved on the Birch and Swinnerton-Dyer conjecture, including the results of $[11,15]$ and [19], can be viewed as part of the larger programme of understanding the modularity of (non-semisimple) $p$-adic Galois representations.

One of the most celebrated modularity results is the statement that all elliptic curves over $\mathbb{Q}$ arise as quotients of suitable modular curves: more precisely, that an elliptic curve $E$ over $\mathbb{Q}$ of conductor $N$ is equipped with a surjective parameterisation

$$
\pi_{E}: X_{0}(N) \longrightarrow E,
$$

where $X_{0}(N)$ is the modular curve attached to Hecke's congruence subgroup $\Gamma_{0}(N)$. This was proved in $[37,40]$, and $[12]$ by showing that the $p$-adic representation

$$
H^{1}(E):=H_{\mathrm{et}}^{1}\left(E_{\overline{\mathbb{Q}}}, \mathbb{Q}_{p}\right)(1)=\left(\lim _{\leftarrow, n} E\left[p^{n}\right]\right) \otimes_{\mathbb{Z}_{p}} \mathbb{Q}_{p}
$$

of $G_{\mathbb{Q}}:=\mathrm{Gal}(\overline{\mathbb{Q}} / \mathbb{Q})$ arises as a quotient of the étale cohomology group ${ }^{1}$

$$
H^{1}\left(X_{0}(N)\right):=H_{\mathrm{et}}^{1}\left(X_{0}(N)_{\overline{\mathbb{Q}}}, \mathbb{Q}_{p}(1)\right) .
$$

The existence of a Galois-equivariant projection

$$
\pi_{E}: H^{1}\left(X_{0}(N)\right) \longrightarrow H^{1}(E)
$$

is the real content of the breakthrough in [40] and [37], the ostensibly stronger geometric version (1) being deduced from it by invoking the Tate conjecture for curves. ${ }^{2}$

Let $E^{\prime}$ be an open subvariety of $E$, i.e. the complement of a zero-dimensional subvariety $\Sigma$ of $E$ over $\mathbb{Q}$. The $p$-adic Galois representation $H^{1}\left(E^{\prime}\right)$ sits in the middle of the short exact excision sequence

$$
0 \longrightarrow H^{1}(E) \longrightarrow H^{1}\left(E^{\prime}\right) \longrightarrow H^{0}(\Sigma)_{0} \longrightarrow 0
$$

of étale cohomology groups, where the subscript of 0 denotes the degree 0 elements of $H^{0}(\Sigma)$. By analogy with (2), the curve $E^{\prime}$ is (provisionally) said to be modular if $H^{1}\left(E^{\prime}\right)$

${ }^{1}$ The systematic shorthand $H^{i}(X):=H_{\mathrm{et}}^{i}\left(X_{\overline{\mathbb{Q}}}, \mathbb{Q}_{p}(i)\right)$ for any variety $X$ over $\mathbb{Q}$ is adopted henceforth to lighten the notations.

${ }^{2}$ Subsequently, (2) has been generalised to a host of other $p$-adic Galois representations, while analogues of (1) remain unavailable in all but the simplest geometric settings. 
arises as a subquotient of $H^{1}(Y)$, where $Y$ is an open sub-Shimura variety of $X_{0}(N)$-the latter being defined, in the style of La Palice, as the complement of a closed sub-Shimura variety.

To completely describe the open sub-Shimura varieties of the modular curve $X_{0}(N)$ over $\mathbb{Q}$, note that the latter is the coarse moduli space of elliptic curves $A$ with a marked subgroup scheme of order $N$, and that its closed sub-Shimura varieties are obtained by imposing additional endomorphism rings, which can only be equal to orders in quadratic imaginary fields. Given such an order $\mathcal{O} \subset K$, the associated closed sub-Shimura variety $\Sigma_{\mathcal{O}} \subset X_{0}(N)$ consists of $C M$ points for $\mathcal{O}$ and is the coarse moduli space of elliptic curves $A$ with level $N$ structure equipped with an optimal embedding $\iota: \mathcal{O} \longrightarrow \operatorname{End}(A)$ (respecting the level structure) and acting in a prescribed way on the cotangent space of $A$. By the theory of complex multiplication, the 0 -dimensional variety $\Sigma_{\mathcal{O}}$ is isomorphic over $K$ (at least, when the discriminant of $\mathcal{O}$ is prime to $N$ ) to $\phi_{K}(N) \operatorname{copies}$ of $\operatorname{spec}\left(H_{\mathcal{O}}\right)$, where $\phi_{K}(N)$ is the number of primitive ideals of $K$ of norm $N$ and $H_{\mathcal{O}}$ is the ring class field of $K$ attached to $\mathcal{O}$, whose Galois group over $K$ is canonically identified with the Picard group of $\mathcal{O}$ via global class field theory.

The complements

$$
Y_{\mathcal{O}}(N):=X_{0}(N)-\Sigma_{\mathcal{O}}
$$

thus provide an exhaustive list of the open sub-Shimura varieties of $X_{0}(N)$. Given the modularity of $E$, the modularity of $E^{\prime}$ amounts to the existence of a Galois-equivariant inclusion

$$
i: H^{0}(\Sigma)_{0} \longrightarrow H^{0}\left(\Sigma_{\mathcal{O}}\right)_{0}
$$

for suitable $\mathcal{O}$, realising $H^{1}\left(E^{\prime}\right)$ as a subquotient of $H^{1}\left(Y_{\mathcal{O}}(N)\right)$ via the pushforward under $\pi_{E}$ and the pullback under $\iota$ of the first row in the following diagram with exact rows:

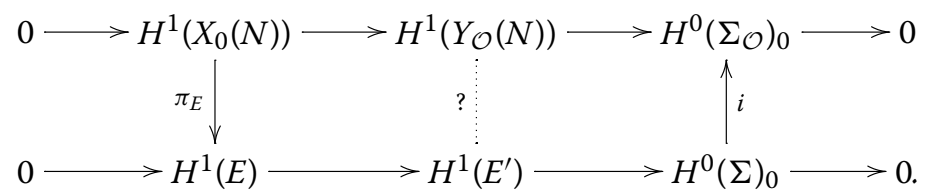

Consider the simplest non-trivial setting where $\Sigma=\left\{P_{1}, P_{2}\right\} \subset E(\mathbb{Q})$ consists of two points defined over $\mathbb{Q}$, so that $H^{0}(\Sigma)_{0}=\mathbb{Q}_{p}$ with trivial Galois action. The resulting extension

$$
0 \longrightarrow H^{1}(E) \longrightarrow H^{1}\left(E^{\prime}\right) \longrightarrow \mathbb{Q}_{p} \longrightarrow 0
$$

encodes the image of the point $P_{2}-P_{1} \in E(\mathbb{Q})$ under the connecting homomorphism

$$
\delta: E(\mathbb{Q}) \longrightarrow H^{1}\left(\mathbb{Q}, H^{1}(E)\right):=\operatorname{Ext}_{G_{\mathbb{Q}}}^{1}\left(\mathbb{Q}_{p}, H^{1}(E)\right)
$$

of Kummer theory, where the Ext group is taken in the category of continuous $p$-adic representations of $G_{\mathbb{Q}}$. The following statement, which gives a "modularity criterion" for $E^{\prime}$ and encapsulates many of the deepest theorems on the Birch and SwinnertonDyer conjecture obtained in the last decades, is of course expected to hold for all elliptic curves $E$, but the reader is cautioned that the proof of the implication (d) $\Rightarrow$ (a) currently requires that $E$ be a semistable elliptic curve having at least one odd prime of non-split multiplicative reduction or at least two odd primes of split multiplicative reduction. 
Theorem 1.1 Assume that the point $P_{2}-P_{1}$ is of infinite order in $E(\mathbb{Q})$. Then the following are equivalent:

(a) The curve $E^{\prime}=E \backslash\left\{P_{1}, P_{2}\right\}$ is modular;

(b) the Hasse-Weil L-series $L(E, s)$ has a simple zero at $s=1$;

(c) the point $P_{2}-P_{1}$ generates $E(\mathbb{Q}) \otimes \mathbb{Q}$ and $\mathbb{I I I}(E / \mathbb{Q})$ is finite;

(d) for all primes $p$, the group $\operatorname{Ext}_{\mathrm{fin}}^{1}\left(\mathbb{Q}_{p}, H^{1}(E)\right)$ of extensions of p-adic representations of the Galois group of $\mathbb{Q}$ that are cristalline at $p$ is one-dimensional over $\mathbb{Q}_{p}$.

Sketch of proof The modularity of $E^{\prime}$ amounts to the statement that there exists an order $\mathcal{O}$ in an imaginary quadratic field $K$ such that the extension (4) can be obtained as the pullback of (3) via an inclusion $i: \mathbb{Q}_{p} \longrightarrow H^{0}\left(\Sigma_{\mathcal{O}}\right)^{G_{\mathbb{Q}}}$, whose image contains a degree 0 divisor

$$
D_{K} \in \operatorname{Div}^{0}\left(\Sigma_{\mathcal{O}}\right)^{G} \subset \operatorname{Div}^{0}\left(X_{0}(N)\right)(\mathbb{Q}) .
$$

This means that the point $P_{1}-P_{2} \in E(\mathbb{Q})$ is a nonzero multiple of the Heegner point $P_{E, K}:=\pi_{E}\left(D_{K}\right)$.

The implication $(\mathrm{a}) \Rightarrow$ (b) therefore follows from the Gross-Zagier formula [21] expressing the height of $P_{E, K}$ as a nonzero multiple of

$$
L^{\prime}(E / K, 1)=L^{\prime}(E, 1) \cdot L\left(E^{K}, 1\right)
$$

where $E^{K}$ is the quadratic twist of $E$ by $K$. The existence of a suitable $K$ for which $L\left(E^{K}, 1\right) \neq 0$ follows from a non-vanishing result of Waldspurger or can be deduced from analytic number theory techniques (cf. [28]).

The implication (b) $\Rightarrow$ (c) was subsequently proved by Kolyvagin [25], who parlayed the non-triviality of $P_{E, K}$ into a bound on the Mordell-Weil rank and the Selmer group of $E$ over $K$.

The implication (c) $\Rightarrow(d)$ is a direct consequence of the definitions: in fact (d) is ostensibly weaker than (c), Selmer groups being less subtle to control than Mordell-Weil and Shafarevich-Tate groups.

The striking implication (d) $\Rightarrow$ (a) follows from Skinner's "converse of the GrossZagier-Kolyvagin Theorem" [33]. This last step is the most recent and combines several new ingredients: the powerful techniques developed by Skinner and Urban to prove the Iwasawa-Greenberg main conjecture for elliptic curves over $\mathbb{Q}$ [35], an important variant explored by Xin Wan in his Ph.D. thesis [39], and the $p$-adic analogue of [21] formulated and proved in [8].

More precisely, choose a prime $p \geq 5$ of good ordinary reduction for $E$ such that $E[p]$ is an irreducible $G_{\mathbb{Q}}$-representation and the image of the restriction map $\operatorname{Sel}_{p}(E) \longrightarrow$ $E\left(\mathbb{Q}_{p}\right) / p E\left(\mathbb{Q}_{p}\right)$ does not lie in the image of $E\left(\mathbb{Q}_{p}\right)[p]$. A result of Waldspurger ensures the existence of an odd quadratic character $\chi$ such that $L(E, \chi, 1) \neq 0$, which can be chosen so that $\chi(2)=\chi(p)=1$. Let $K$ denote the imaginary quadratic field associated to $\chi$. The $p$-adic Selmer group Ext ${ }_{K \text { fin }}^{1}\left(\mathbb{Q}_{p}, H^{1}(E)\right)$ of $E$ over $K$ (defined as an Ext group in the category of cristalline representations of $G_{K}$ ) decomposes as a direct sum of eigenspaces

$$
\operatorname{Ext}_{K, \text { fin }}^{1}\left(\mathbb{Q}_{p}, H^{1}(E)\right) \simeq \operatorname{Ext}_{\text {fin }}^{1}\left(\mathbb{Q}_{p}, H^{1}(E)\right) \oplus \operatorname{Ext}_{K, \text { fin }}^{1}\left(\mathbb{Q}_{p}, H^{1}(E)\right)^{-}
$$

with respect to the action of complex conjugation. Because $L(E, \chi, 1) \neq 0$, the results of Kolyvagin (or of Kato) imply the triviality of $\operatorname{Ext}_{K, \mathrm{fin}}^{1}\left(\mathbb{Q}_{p}, H^{1}(E)\right)^{-}$. Assumption (d) 
therefore implies that Ext ${ }_{K, \text { fin }}^{1}\left(\mathbb{Q}_{p}, H^{1}(E)\right)$ is one-dimensional over $\mathbb{Q}_{p}$. One can then argue as in [33]. Namely, the running hypotheses ensure that both Lemma 2.3.2 and Proposition 2.7.3 of loc.cit. apply, and hence, that a $p$-adic $L$-function of the type that occurs in [39] and [8] (which interpolates critical values of the $L$-series of the Rankin convolution of the modular form $f$ associated to $E$ with suitable Hecke characters of $K$ of higher infinity-type) does not vanish at the trivial point, which lies outside its region of classical interpolation. This in turn implies, in the light of [33, Corollary 2.6.2] resting on the variant of the Gross-Zagier formula of [8], that the Heegner point $P_{E, K}$ has non-trivial $p$-adic formal group logarithm and is therefore non-torsion. As already explained, the non-triviality of $P_{E, K}$ is equivalent to (a), and the implication $(d) \Rightarrow(a)$ follows.

The Birch and Swinnerton-Dyer conjecture admits an extension to elliptic curves twisted by Artin representations which arises very naturally in the context of the modularity questions framed above. Let

$$
\varrho: \operatorname{Gal}(H / \mathbb{Q}) \hookrightarrow \operatorname{Aut}\left(V_{\varrho}\right) \simeq \mathrm{GL}_{n}\left(\overline{\mathbb{Q}}_{p}\right)
$$

be an $n$-dimensional representation of the Galois group of a finite extension $H / \mathbb{Q}$, a socalled Artin representation, viewed as having coefficients in $\overline{\mathbb{Q}}_{p}$. The pair $(E, \varrho)$ gives rise to the Hasse-Weil-Artin $L$-series

$$
L(E, \varrho, s):=\prod_{\ell} \operatorname{det}\left(1-\ell^{-s}\left(\mathrm{Fr}_{\ell}^{-1}\right)_{\left(H^{1}(E) \otimes V_{\varrho}\right)^{I_{\ell}}}\right)^{-1},
$$

where the product is taken over the rational primes $\ell$, the arithmetic frobenius element at $\ell$ is denoted by $\mathrm{Fr}_{\ell}$, and $I_{\ell}$ denotes the inertia group at $\ell$. The equivariant Birch and Swinnerton-Dyer conjecture for $E$ and $\varrho$, denoted $\operatorname{BSD}(E, \varrho)$, asserts that

$$
\operatorname{ord}_{s=1} L(E, \varrho, s)=\operatorname{dim}_{\overline{\mathbb{Q}}_{p}}\left(E(H) \otimes V_{\varrho}\right)^{G_{\mathbb{Q}}} .
$$

As a first step to understanding $\operatorname{BSD}(E, \varrho)$, it is natural to ask which $\kappa \in \operatorname{Ext}_{\text {fin }}^{1}\left(V_{\varrho}, H^{1}(E)\right)$ can be realised as a subquotient of a suitable $H^{1}\left(Y_{\mathcal{O}}(N)\right)$. The Artin representation $H^{0}\left(\Sigma_{\mathcal{O}}\right)_{0}$ which appears in the upper rightmost term of the diagram (3) is readily analysed using the theory of complex multiplication. Namely, the slightly larger Artin representation $H^{0}\left(\Sigma_{\mathcal{O}}\right)$ decomposes as a direct sum

$$
\begin{gathered}
H^{0}\left(\Sigma_{\mathcal{O}}\right) \otimes \overline{\mathbb{Q}}_{p}=\oplus_{j=1}^{\phi_{K}(N)} W_{j}, \quad \text { where } W_{j}=\oplus_{\psi} V_{j}(\psi), \\
\text { with } V_{j}(\psi) \subset V_{\psi}:=\operatorname{Ind}_{K}^{\mathbb{Q}} \psi .
\end{gathered}
$$

In this equation, the second direct sum is taken over the non-trivial, $\overline{\mathbb{Q}}_{p}$-valued, finite order characters $\psi$ of $\mathrm{Gal}\left(H_{\mathcal{O}} / K\right)$ modulo the involution $\psi \mapsto \psi^{-1}$, and $V_{j}(\psi)$ is a non-trivial irreducible constituent of the two-dimensional representation $V_{\psi}$ obtained by inducing the Galois character $\psi$ from $G_{K}$ to $G_{\mathbb{Q}}$. The representation $V_{\psi}$ is irreducible precisely when $\psi \neq \psi^{-1}$, and in this case a non-trivial class $\kappa \in \operatorname{Ext}_{\text {fin }}^{1}\left(V_{\psi}, H^{1}(E) \otimes \overline{\mathbb{Q}}_{p}\right)$ is expected to be modular if and only if (any of) the analogues of conditions (b)-(d) of Theorem 1.1 are satisfied, namely:

(b') The Hasse-Weil-Artin $L$-series $L\left(E, V_{\psi}, s\right)$ has a simple zero at $s=1$;

(c') the representation $V_{\psi}$ occurs with multiplicity one in $E(H) \otimes \overline{\mathbb{Q}}_{p}$, and the $V_{\psi}$-isotypic component of the $\mathbb{I I}(E / H)$ is finite;

(d') the group $\operatorname{Ext}_{\text {fin }}^{1}\left(V_{\psi}, H^{1}(E) \otimes \overline{\mathbb{Q}}_{p}\right)$ is one-dimensional over $\overline{\mathbb{Q}}_{p}$, and generated by $\kappa$. 
Although such a precise result does not seem to appear in the literature, all the ingredients needed to prove it seem to be available in principle.

The rather narrow notion of modularity described above has a few visible drawbacks:

(1) Very few Artin representations arise in the cohomology of the 0-dimensional Shimura varieties $\Sigma_{\mathcal{O}}$, which are not even rich enough to capture all of the irreducible two-dimensional Artin representations of $\mathbb{Q}$. The open Shimura varieties $Y_{\mathcal{O}}(N)$ thus appear to give no purchase on $\operatorname{BSD}(E, \varrho)$ when $\varrho$ is not induced from a ring class character of an imaginary quadratic field.

(2) Theorem 1.1 suggests that the modularity of elements of $\operatorname{Ext}_{\mathrm{fin}}^{1}\left(V_{\psi}, H^{1}(E)\right)$ is purely a "rank one phenomenon": if this Ext group has dimension $>1$, none of its elements are expected to be realised in subquotients of any $H^{1}\left(Y_{\mathcal{O}}(N)\right)$.

In order to relate a larger class of non-semisimple Galois representations to modular forms, it becomes desirable to relax the notion of modularity. One way in which one might try to do this is by replacing the curves $Y_{\mathcal{O}}(N)$ with more general "open Shimura varieties". These should include all the varieties whose cohomology (at least, after semisimplification) is directly related to automorphic forms via a suitable generalisation of the Eichler-Shimura congruence, and would eventually encompass the complements of sub-Shimura varieties in larger Shimura varieties, as well as Kuga-Sato varieties and other natural varieties fibred over Shimura varieties, the complements of Heegner cycles in such varieties, and so on. With this expanded notion of modularity, the programme of characterising the nonsemisimple Galois representations that are modular becomes richer and more subtle. See [9] for a fragment of experimental mathematics that might be viewed as fitting into this programme. The following question seems like it might repay further investigation, given the paucity of evidence, both theoretical and experimental, that has been gathered around it so far:

Question 1.2 Let $V_{1}$ and $V_{2}$ be Galois representations for which hom $\left(V_{1}, V_{2}\right)$ is irreducible. Suppose that there is a non-trivial $\kappa \in \operatorname{Ext}_{\text {fin }}^{1}\left(V_{1}, V_{2}\right)$ arising as a subquotient of the cohomology of an open Shimura variety. Is $\operatorname{Ext}_{\text {fin }}^{1}\left(V_{1}, V_{2}\right)$ necessarily one-dimensional?

If the answer to this question were "yes", it would imply that the open curve $E-\left\{P_{1}, P_{2}\right\}$ discussed in Theorem 1.1 is never modular when $\operatorname{rank}(E(\mathbb{Q}))>1$. (But see the inspiring article [29], as well as the striking ongoing work of Zhiwei Yun and Wei Zhang in the function field case, for some tantalising ideas in the opposite, more optimistic direction.)

A second idea for enlarging the class of $p$-adic Galois representations deemed to be modular is to allow $p$-adic limits of Galois representations arising in the cohomology of (open) Shimura varieties. This idea is very natural in the light of the classical work of Deligne-Serre on Artin representations attached to weight one forms, whereby such Artin representations are obtained by piecing together the Galois representations attached to modular forms of higher weights which are realised in the cohomology of KugaSato varieties. It is via this broader notion of modularity that all odd, irreducible twodimensional Artin representations of $\mathbb{Q}$ can be related to modular forms. The idea of realising automorphic Galois representations as $p$-adic limits has become pervasive in the subject, and led to important advances: for example, it plays a key role in the recent construction [22] by Harris, Lan, Taylor, and Thorne of Galois representations attached to non-self-dual automorphic forms on $\mathrm{GL}_{n}$. Even more germane to this article, $p$-adic limits 
of automorphic Galois representations appear to capture non-trivial extension classes going beyond settings of "multiplicity one", as is illustrated by the following theorem of Skinner and Urban [34, Thm. B]:

Theorem 1.3 Let $E$ be an elliptic curve over $\mathbb{Q}$. If $L(E, s)$ vanishes to even order $\geq 2$ ats $=1$, then the Selmer group $\operatorname{Ext}_{\mathrm{fin}}^{1}\left(\mathbb{Q}_{p}, H^{1}(E)\right)$ of E contains at least two linearly independent modular classes.

The modular classes in this theorem are constructed as $p$-adic limits of geometric Galois representations in the cohomology of Shimura varieties associated to the unitary group $U(2,2)$. Although these geometric Galois representations are believed to be semisimple, Theorem 1.3 rests on the fact that this feature need not persist in the limit.

The primary goal of this article is to discuss a different approach for constructing canonical extension classes of $\varrho$ by $H^{1}(E)$ for a large class of self-dual Artin representations $\varrho$ of dimension 4 (and their lower-dimensional subrepresentations, in case $\varrho$ is reducible) arising as the tensor product $\varrho=\varrho_{1} \otimes \varrho_{2}$ of a pair of odd, two-dimensional Artin representations. The construction of these classes is one of the main results of [19] (resp. [11]) when both $\varrho_{1}$ and $\varrho_{2}$ are irreducible (resp. when exactly one of $\varrho_{1}$ and $\varrho_{2}$ is irreducible), and is based on $p$-adic limits of non-semisimple, but "geometrically modular" Galois representations. These limit classes are referred to as generalised Kato classes because their construction is inspired by the seminal work [23] of Kato (cf. also [6,32]) on $\operatorname{BSD}(E, \chi)$ for $\chi$ a Dirichlet character. Like Heegner points in the setting of $\operatorname{BSD}\left(E, V_{\psi}\right)$, generalised Kato classes enjoy close relations to ( $p$-adic) Hasse-Weil-Artin $L$-functions attached to $E$ and $\varrho$, but unlike Heegner points, they are expected to generate a non-trivial subgroup of the Selmer group attached to $E$ and $\varrho$ precisely when $\operatorname{ord}_{s=1} L(E, \varrho, s)=2$. The formulae of [19] (cf. Corollary 3.6 below) relating the linear independence of two generalised Kato classes to the non-vanishing of certain $p$-adic $L$-series can thus be regarded as a $p$-adic Gross-Zagier formula "in analytic rank two".

The main new contribution of this article is a conjecture expressing the same generalised Kato classes as canonical elements in $\left(E(H) \otimes V_{\varrho}\right)^{G_{\mathbb{Q}}}$ when this latter space is two-dimensional. This conjecture strengthens and refines the "elliptic Stark conjecture" of [15], and provides a framework for understanding the results of [11,15] and [19]. The settings in which $\varrho$ is reducible often take on special arithmetic interest and are described in detail in the last chapter.

\section{Hida families and periods for weight one forms}

This section provides background on certain canonical structures associated to a weight one form $g$, arising from the Hida families specialising in weight one to (a $p$-stabilisation of) $g$. These are important for the conjectures of Sect. 3.4, but Sect. 2 can be skipped on a first reading by the reader wishing to get a quick feeling for the generalised Kato classes described in Sects. 3.1 and 3.2. On the other hand, it is also worth noting that Sect. 2 is entirely self-contained. Conjecture 2.1 , which can be viewed as a $p$-adic analogue of the Stark conjecture for the adjoint of the Galois representation attached to a weight one form, appears to be new and may be of independent interest.

Let $g \in S_{1}(N, \chi)$ be a newform of weight one and level $N$ with Fourier coefficients in a field $L$, and let 


$$
\varrho: G_{\mathbb{Q}} \longrightarrow \operatorname{Aut}(V) \simeq \mathrm{GL}_{2}(L)
$$

be the Artin representation associated to it by the construction of Deligne and Serre. We view $\varrho$ as acting on a two-dimensional $L$-vector space $V$, where $L \subset \mathbb{C}$ can be chosen to be contained in a cyclotomic field.

Let $H$ be the number field cut out by $\varrho$, so that $\varrho$ factors through $G a l(H / \mathbb{Q})$. Fix a rational prime $p$ and choose a prime $\mathfrak{p}$ of $H$ above $p$. The latter determines a canonical inclusion

$$
H \subset H_{p} \subset \overline{\mathbb{Q}}_{p}
$$

of $H$ in its completion $H_{p}$ at $\mathfrak{p}$. Assume that the pair $(\varrho, p)$ satisfies the following conditions:

(I) The prime $p$ splits completely in $L / \mathbb{Q}$, so that $L$ is equipped with an embedding into $\mathbb{Q}_{p}$ which will be fixed from now on. This assumption, which is made solely to lighten the notations and could easily be dispensed with, allows $\varrho$ to be viewed as a $\mathbb{Q}_{p}$-linear representation via the natural action of $G_{\mathbb{Q}}$ on the $\mathbb{Q}_{p}$-vector space $V \otimes_{L} \mathbb{Q}_{p}$

(II) The representation $V$ is unramified at $p$. There is then a well-defined arithmetic frobenius element

$$
\operatorname{Fr}_{p} \in \operatorname{Gal}(H / \mathbb{Q})
$$

acting canonically on $V$, and the characteristic polynomial of $\varrho\left(\mathrm{Fr}_{p}\right)$ is equal to the Hecke polynomial

$$
x^{2}-a_{p}(g) x+\chi(p)=:\left(x-\alpha_{g}\right)\left(x-\beta_{g}\right)
$$

attached to $g$.

(III) The modular form $g$ is regular at $p$, i.e. $\alpha_{g} \neq \beta_{g}$. After possibly enlarging $L$, it may also be assumed that this coefficient field contains the roots of unity $\alpha_{g}$ and $\beta_{g}$.

(IV) The representation $\varrho_{g}$ is not induced from a character of a real quadratic field $K$ in which the prime $p$ splits. The rationale for this condition, which seems to be essential for a number of the constructions and conjectures proposed in this paper, is explained in $[15, \mathbb{\$} 1.1]$.

The $p$-stabilisations of $g$ at $p$ are the normalised eigenforms of weight one with Fourier coefficients in $L$ defined by

$$
g_{\alpha}:=g(z)-\beta_{g} g(p z), \quad g_{\beta}:=g(z)-\alpha_{g} g(p z) .
$$

They are eigenvectors for the $U_{p}$-operator satisfying

$$
U_{p} g_{\alpha}=\alpha_{g} g_{\alpha}, \quad U_{p} g_{\beta}=\beta_{g} g_{\beta} .
$$

The Artin representation $V$ decomposes naturally as a direct sum

$$
V=V^{\alpha} \oplus V^{\beta}
$$

into one-dimensional eigenspaces for $\mathrm{Fr}_{p}$, with eigenvalues $\alpha_{g}$ and $\beta_{g}$, respectively.

By a theorem of Hida, there exists a finite flat extension $\Lambda_{g}$ of the Iwasawa algebra $\Lambda$ and a Hida family $\underline{g} \in \Lambda_{\underline{g}}[[q]]$ of tame level $N$ and tame character $\chi$ passing through the $p$ stabilised weight one eigenform $g_{\alpha}$. When $g$ is cuspidal, the regularity hypothesis imposed on $g$ implies that such a Hida family is unique, thanks to a recent result of Bellaïche and Dimitrov [1]. 
The Hida family $g$ comes equipped with the following canonical structures:

(a) There is a locally free $\Lambda_{g}$-module $\mathbb{V}_{g}$ of rank two, affording Hida's ordinary $\Lambda$-adic Galois representation

$$
\varrho_{\underline{g}}: G_{\mathbb{Q}} \longrightarrow \operatorname{Aut}_{\Lambda_{g}}\left(\mathbb{V}_{g}\right)
$$

which is realised in the inverse limit of ordinary étale cohomology groups associated to the tower $X_{1}\left(N p^{r}\right)$ of modular curves. This representation interpolates the Galois representations associated by Deligne to the classical specialisations of $\underline{g}$.

(b) The restriction of $\mathbb{V}_{g}$ to $G_{\mathbb{Q}_{p}}$ admits a stable filtration

$$
0 \longrightarrow \mathbb{U}_{g} \longrightarrow \mathbb{V}_{g} \longrightarrow \mathbb{W}_{g} \longrightarrow 0,
$$

where both $\mathbb{U}_{g}$ and $\mathbb{W}_{g}$ are flat $\Lambda_{g}\left[G_{\mathbb{Q}_{p}}\right]$-modules that are locally free of rank one over $\Lambda_{g}$, and the quotient $\mathbb{W}_{g}$ is unramified, with $\operatorname{Fr}_{p}$ acting on $\mathbb{W}_{g}$ as multiplication by the $p$-th Fourier coefficient $a_{p}(\underline{g})$.

(c) Let $\mathbb{Q}_{p}^{\mathrm{nr}}$ denote the maximal unramified extension of $\mathbb{Q}_{p}$ and let $\widehat{\mathbb{Q}}_{p}^{\mathrm{nr}}$ denote its $p$-adic completion. In [30], Ohta constructs a canonical $\Lambda_{g}$-adic period

$$
\omega_{\underline{g}} \in D\left(\mathbb{W}_{g}\right):=\left(\widehat{\mathbb{Q}}_{p}^{\mathrm{nr}} \hat{\otimes} \mathbb{W}_{g}\right)^{G_{\mathbb{Q} p}},
$$

corresponding to the normalised $\Lambda$-adic eigenform $\underline{g}$ under the isomorphism in Theorem (A) of the introduction of [30].

(d) There is a natural perfect Galois-equivariant duality, given in Theorem (B) of the introduction of [30],

$$
\mathbb{U}_{g} \times \mathbb{W}_{g} \longrightarrow \Lambda_{g}\left(\operatorname{det}\left(\varrho_{g}\right)\right)
$$

where $G_{\mathbb{Q}}$ acts on the module $\Lambda_{g}$ of the right-hand side via the determinant of $\varrho_{g}$.

Let

$$
y_{g}: \Lambda_{g} \longrightarrow \mathbb{Q}_{p}
$$

be the specialisation map attached to the $p$-stabilised weight one form $g_{\alpha}$. By specialising the structures above attached to $g$ via the map $y_{g}$, we obtain

(a') A non-canonical isomorphism of $\mathbb{Q}_{p}\left[G_{\mathbb{Q}}\right]$-modules

$$
\Phi_{g_{\alpha}}: V_{g}:=\mathbb{V}_{g} \otimes_{y_{g}} \mathbb{Q}_{p} \stackrel{\sim}{\longrightarrow} V \otimes_{L} \mathbb{Q}_{p}
$$

(b') A non-trivial $G_{\mathbb{Q}_{p}}$-stable filtration

$$
0 \longrightarrow U_{g} \longrightarrow V_{g} \longrightarrow W_{g} \longrightarrow 0
$$

of $V_{g}$ by one-dimensional subspaces, where $U_{g}:=\mathbb{U}_{g} \otimes_{y_{g}} \mathbb{Q}_{p}$ and $W_{g}:=\mathbb{W}_{g} \otimes_{y_{g}} \mathbb{Q}_{p}$. The Frobenius element $\operatorname{Fr}_{p}$ acts on $W_{g}$ and $U_{g}$ as multiplication by $\alpha_{g}$ and $\beta_{g}$, respectively. Since these eigenvalues are assumed to be distinct, the exact sequence above splits canonically, leading to the identifications

$$
U_{g}=V_{g}^{\beta}, \quad W_{g}=V_{g}^{\alpha}, \quad V_{g}=U_{g} \oplus W_{g}=V_{g}^{\beta} \oplus V_{g}^{\alpha} .
$$

(c') Specialising Ohta's period leads to a canonical element

$$
\omega_{g_{\alpha}}:=y_{g}\left(\omega_{\underline{g}}\right) \in D\left(V_{g}^{\alpha}\right):=\left(\mathbb{Q}_{p}^{\mathrm{nr}} \otimes V_{g}^{\alpha}\right)^{G_{\mathbb{Q}_{p}}}=\left(H_{p} \otimes V_{g}^{\alpha}\right)^{G_{\mathbb{Q} p}} .
$$


(d') The duality in (d) above specialises via $y_{g}$ to a canonical pairing of $\mathbb{Q}_{p}$-vector spaces

$$
\langle,\rangle: V_{g}^{\beta} \times V_{g}^{\alpha} \longrightarrow \mathbb{Q}_{p}(\chi),
$$

which induces a pairing by functoriality (denoted by the same symbol by a slight abuse of notation):

$$
\langle,\rangle: D\left(V_{g}^{\beta}\right) \times D\left(V_{g}^{\alpha}\right) \longrightarrow D\left(\mathbb{Q}_{p}(\chi)\right) .
$$

When this pairing is perfect, it can be used to define a period $\eta_{g_{\alpha}} \in D\left(V_{g}^{\beta}\right)$, as the unique element satisfying

$$
\left\langle\eta_{g_{\alpha}}, \omega_{g_{\alpha}}\right\rangle=\mathfrak{g}(\chi) \otimes 1,
$$

where

$$
\mathfrak{g}(\chi):=\sum_{j=1}^{\left.\mathfrak{f}_{\chi}\right)} \chi(j) e^{2 \pi i j / \mathfrak{f}_{\chi}}
$$

is the Gauss sum attached to the Dirichlet character $\chi$, viewed as an element of $H_{p}$ by assigning an $\mathfrak{f}_{\chi}$-th root of unity in $H_{p}$ to the complex number $e^{2 \pi i / \mathfrak{f}_{\chi}}$.

Making use of the above arsenal we now turn to introduce certain $p$-adic periods associated to $g$ and the choice of a $L$-structure on $V_{g}$. We assume for simplicity in the sequel that $g$ is a cusp form, and thus $V_{g}$ is irreducible.

Fix a $G_{\mathbb{Q}}$-equivariant isomorphism $j_{g}: V \otimes_{L} \mathbb{Q}_{p} \longrightarrow V_{g}$ and let $V_{g}^{L}:=j_{g}(V)$ denote the associated $L$-rational structure on $V_{g}$, which by Schur's lemma is well defined up to scaling by $\mathbb{Q}_{p}^{\times}$. Since $j_{g}$ induces isomorphisms $V_{g}^{\alpha} \simeq V^{\alpha} \otimes_{L} \mathbb{Q}_{p}$ and $V_{g}^{\beta} \simeq V^{\beta} \otimes_{L} \mathbb{Q}_{p}$, we may choose $L$-bases $v_{g}^{\alpha}$ and $v_{g}^{\beta}$ for $V_{g}^{L} \cap V_{g}^{\alpha}$ and $V_{g}^{L} \cap V_{g}^{\beta}$, respectively, so that

$$
V_{g}^{L} \cap V_{g}^{\alpha}=\left\langle v_{g}^{\alpha}\right\rangle_{L} \quad \text { and } \quad V_{g}^{L} \cap V_{g}^{\beta}=\left\langle v_{g}^{\beta}\right\rangle_{L} .
$$

Define $p$-adic periods

$$
\Omega_{g_{\alpha}}=\Omega_{g_{\alpha}}\left(V_{g}^{L}\right) \in H_{p}^{\mathrm{Fr}_{p}=\alpha_{g}^{-1}}, \quad \Xi_{g_{\alpha}}=\Xi_{g_{\alpha}}\left(V_{g}^{L}\right) \in H_{p}^{\mathrm{Fr}_{p}=\beta_{g}^{-1}}
$$

by setting

$$
\Omega_{g_{\alpha}} \otimes v_{g}^{\alpha}=\omega_{g_{\alpha}}, \quad \Xi_{g_{\alpha}} \otimes v_{g}^{\beta}=\eta_{g_{\alpha}} .
$$

These periods depend on the choice of the basis $\left(v_{g}^{\alpha}, v_{g}^{\beta}\right)$ for $V_{g}^{L}$, but only up to multiplication by $L^{\times}$.

Furthermore, for all $\mu \in \mathbb{Q}_{p}^{\times}$,

$$
\Omega_{g_{\alpha}}\left(\mu V_{g}^{L}\right)=\mu^{-1} \cdot \Omega_{g_{\alpha}}\left(V_{g}^{L}\right), \quad \Xi_{g_{\alpha}}\left(\mu \cdot V_{g}^{L}\right)=\mu^{-1} \cdot \Xi_{g_{\alpha}}\left(V_{g}^{L}\right) .
$$

It follows that the ratio

$$
\mathcal{L}_{g_{\alpha}}:=\frac{\Omega_{g_{\alpha}}}{\Xi_{g_{\alpha}}} \in\left(H_{p}\right)^{\operatorname{Fr}_{p}=\frac{\beta_{g}}{\alpha_{g}}}
$$

is a number in $H_{p}^{\times}$that is well defined up to multiplication by elements in $L^{\times}$.

This expression is a canonical $p$-adic period attached to the eigenform $g_{\alpha}$ and can be viewed as a $p$-adic avatar of the Petersson norm of $g$.

Definition 1.8 of $[15, \$ 1.2]$ associates in many cases a canonical $p$-adic Stark unit $u_{g_{\alpha}}$ attached to $g_{\alpha}$ as follows. Let $V_{\text {ad }}:=\operatorname{End}_{0}(V)$ be the three-dimensional adjoint representa- 
tion attached to $V$ consisting of trace zero endomorphisms of $V$. Since complex conjugation acts with eigenvalues $-1,-1$, and 1 on $V_{\text {ad }}$, it follows that hom $G_{\mathbb{Q}}\left(V_{\text {ad }},\left(\mathcal{O}_{H}^{\times}\right)_{L}\right)=L \cdot \varphi_{\text {ad }}$ for a suitable generator $\varphi_{\mathrm{ad}}$. If one further assumes that $\alpha_{g} \neq \pm \beta_{g}$, then the subspace of $V_{\text {ad }}$ on which $\operatorname{Fr}_{p}$ acts as multiplication by $\frac{\beta_{g}}{\alpha_{g}}$ is one-dimensional; after choosing an $L$-basis $v_{g_{\alpha}}$ for it, one lets

$$
u_{g_{\alpha}}:=\varphi_{\mathrm{ad}}\left(v_{g_{\alpha}}\right) .
$$

This element is well defined up to multiplication by $L^{\times}$, and hypothesis (IV) above guarantees that it is a nonzero vector of the $V_{\text {ad }}$-isotypic subspace of $\left(\mathcal{O}_{H}^{\times}\right)_{L}$. See [15, $\left.\$ 1.2\right]$ for further details.

Conjecture 2.1 The period in (9) satisfies

$$
\mathcal{L}_{g_{\alpha}}=\log _{p}\left(u_{g_{\alpha}}\right) \quad\left(\bmod L^{\times}\right) .
$$

Remark 2.2 It would be interesting to test this conjecture numerically. To the extent that $\mathcal{L}_{g_{\alpha}}$ is a $p$-adic avatar of the Petersson norm of $g$, Conjecture 2.1 can be viewed as a $p$-adic analogue of the Stark conjecture for the $L$-function attached to the adjoint of $g$, in the form in which it is illustrated, for example, in the concluding paragraphs of [36].

\section{Generalised Kato classes}

\subsection{Definition}

Let $E$ be an elliptic curve over $\mathbb{Q}$ and let

$$
\varrho_{1}, \varrho_{2}: \mathrm{Gal}(H / \mathbb{Q}) \longrightarrow \mathrm{GL}_{2}(L)
$$

be odd, irreducible two-dimensional Artin representations of $G_{\mathbb{Q}}$ satisfying

$$
\chi:=\operatorname{det}\left(\varrho_{1}\right)=\operatorname{det}\left(\varrho_{2}\right)^{-1},
$$

where $L$ and $H$ are finite extensions of $\mathbb{Q}$ (and $L$ is chosen, as before, to be contained in a cyclotomic field). Let $V_{1}$ and $V_{2}$ be $L[\mathrm{Gal}(H / \mathbb{Q}]$-modules which are two-dimensional over $L$ and realise $\varrho_{1}$ and $\varrho_{2}$, respectively. Observe that

$$
V_{12}:=V_{1} \otimes_{L} V_{2}
$$

is a four-dimensional $L$-linear representation of $\operatorname{Gal}(H / \mathbb{Q})$ with real traces, i.e. it is isomorphic to its contragredient representation.

Fix a rational prime $p$ and continue to assume that hypotheses (I-IV) of the previous section hold for both the pairs $\left(\varrho_{1}, p\right)$ and $\left(\varrho_{2}, p\right)$.

The progress in modularity realised over the last two decades implies the existence of cusp forms $f, g$, and $h$ attached to $E, \varrho_{1}$, and $\varrho_{2}$, respectively, whose associated $p$-adic representations, denoted $V_{f}, V_{g}$ and $V_{h}$, satisfy

$$
H^{1}(E)=V_{f}, \quad V_{1} \otimes_{L} \mathbb{Q}_{p} \simeq V_{g}, \quad V_{2} \otimes_{L} \mathbb{Q}_{p} \simeq V_{h} .
$$

It is important to keep in mind that the last two isomorphisms, whose existence is proved by comparing traces on both sides, are only well defined up to multiplication by a scalar in $\mathbb{Q}_{p}^{\times}$(by Schur's lemma), and that the $\mathbb{Q}_{p}$-vector spaces $V_{g}$ and $V_{h}$ therefore admit no natural $L$-rational structure. Let 


$$
V_{g h}:=V_{g} \otimes V_{h}, \quad V_{f g h}:=V_{f} \otimes V_{g h}
$$

denote the tensor products of $\mathbb{Q}_{p}$-linear representations of $G_{\mathbb{Q}}$, and write

$$
j_{g h}: V_{12} \otimes_{L} \mathbb{Q}_{p} \longrightarrow V_{g h}
$$

for the isomorphism induced from (11). Let

$$
V_{g h}^{L}:=j_{g h}\left(V_{12}\right)
$$

denote the resulting $G_{\mathbb{Q}}$-stable $L$-rational structure on $V_{g h}$, which is well defined up to multiplication by a scalar in $\mathbb{Q}_{p}^{\times}$, even when $V_{g h}$ is reducible, because $V_{g}$ and $V_{h}$ themselves are irreducible.

Because of (11), the Hasse-Weil and Artin $L$-functions attached to $E, \varrho_{1}$, and $\varrho_{2}$ are equal to the Hecke $L$-functions attached to $f, g$, and $h$, respectively:

$$
L(E, s)=L(f, s), \quad L\left(\varrho_{1}, s\right)=L(g, s), \quad L\left(\varrho_{2}, s\right)=L(h, s),
$$

and therefore admit functional equations and analytic continuations to the entire complex plane. By the theory of Rankin-Selberg and Garrett, the same is true of the degree $8 \mathrm{~L}$ function $L\left(V_{f g h}, s\right)$ attached to the convolution of $f, g$, and $h$.

Let $N=\operatorname{lcm}\left(N_{f}, N_{g}, N_{h}\right)$ denote the least common multiple of the conductors of $E, \varrho_{1}$, and $\varrho_{2}$ and assume further that $p$ does not divide $N$. As in the previous section, let

$$
\begin{aligned}
& x^{2}-a_{p}(g) x+\chi(p)=:\left(x-\alpha_{g}\right)\left(x-\beta_{g}\right), \\
& \quad x^{2}-a_{p}(h) x+\chi^{-1}(p)=:\left(x-\alpha_{h}\right)\left(x-\beta_{h}\right)
\end{aligned}
$$

be the Hecke polynomials at $p$ attached to $g$ and $h$, respectively, and assume that the coefficient field $L$ contains the roots of unity $\alpha_{g}, \beta_{g}, \alpha_{h}$, and $\beta_{h}$. Denote as before by

$$
\begin{aligned}
g_{\alpha} & :=g(z)-\beta_{g} g(p z), \quad g_{\beta}:=g(z)-\alpha_{g} g(p z), \quad h_{\alpha}:=h(z)-\beta_{h} h(p z), \\
& h_{\beta}:=h(z)-\alpha_{h} h(p z)
\end{aligned}
$$

the relevant $p$-stabilisations of $g$ and $h$.

One of the running assumptions of [19] that is also enforced in this article is that the Artin conductor of $V_{g h}$ is relatively prime to the conductor of $E$. Under this assumption, Prasad [31, Theorem 1.4] implies that the local root numbers that govern the sign in the functional equation for $L\left(E, V_{g h}, s\right)$ are equal to 1 at all places of $\mathbb{Q}$, and the Hasse-WeilArtin $L$-function attached to $E$ and $V_{g h}$ therefore vanishes to even order at the symmetry point $s=1$ for its functional equation.

The article [19] describes the construction of four canonical (a priori non-trivial, and distinct) generalised Kato classes

$$
\kappa\left(f, g_{\alpha}, h_{\alpha}\right), \kappa\left(f, g_{\alpha}, h_{\beta}\right), \kappa\left(f, g_{\beta}, h_{\alpha}\right), \kappa\left(f, g_{\beta}, h_{\beta}\right) \in H^{1}\left(\mathbb{Q}, V_{f g h}\right) .
$$

These classes are essentially obtained as $p$-adic limits

$$
\kappa\left(f, g_{\alpha}, h_{\alpha}\right):=\lim _{k \rightarrow 1} \kappa\left(f, g_{k}, h_{k}\right),
$$

as $\left(g_{k}, h_{k}\right)$ range over the classical specialisations of weight $k \geq 2$ of Hida families $g$ and $\underline{h}$ specialising to $g_{\alpha}$ and $h_{\alpha}$, respectively, in weight one, and $\kappa\left(f, g_{k}, h_{k}\right)$ arises from 
a geometric construction whereby it is realised in the $p$-adic étale cohomology of some (open) variety over $\mathbb{Q}$.

More precisely, let $V_{f}(N)$ denote the $f$-isotypic component of $H^{1}\left(X_{0}(N)\right.$ ), which is (noncanonically) isomorphic to a finite number of copies of $V_{f}$, indexed by the positive divisors of $N / N_{f}$. Let $V_{g}(N)$ and $V_{h}(N)$ denote the similar spaces occurring as the weight one specialisations of the $g$ and $\underline{h}$-isotypic parts of the inverse limits of the ordinary quotients of $H^{1}\left(X_{1}\left(N p^{s}\right)\right.$ ), which are abstractly isomorphic to a direct sum of finitely many copies of $V_{g}$ and $V_{h}$, respectively, endowed with all the structures described in (a')-(d') of Sect. 2. The classes in (14) of [19] take values in the Galois representation

$$
V_{f g h}(N)=V_{f}(N) \otimes V_{g}(N) \otimes V_{h}(N)
$$

and the classes of (14) are obtained by applying to them a suitable surjective $G_{\mathbb{Q}^{-}}$ equivariant projection

$$
\pi: V_{f g h}(N) \longrightarrow V_{f g h}
$$

compatible with the $L$-structure, filtration, Ohta periods, and dualities described in (a')(d'). The dependence of $\kappa\left(f, g_{\alpha}, h_{\alpha}\right)$ on the choice of $\pi$ is suppressed from the notations but should be kept in mind.

The generalised Kato classes belong to the global cohomology group $H^{1}\left(\mathbb{Q}, V_{f g h}\right)=$ $\operatorname{Ext}_{G_{\mathbb{Q}}}^{1}\left(\mathbb{Q}_{p}, V_{f g h}\right)$, where $\mathbb{Q}_{p}$ stands for the one-dimensional $p$-adic representation of $G_{\mathbb{Q}}$ with trivial action and the Ext group is taken in the category of finite-dimensional $\mathbb{Q}_{p}$ vector spaces equipped with a continuous $G_{\mathbb{Q}^{-}}$-action (whose restriction to $G_{\mathbb{Q}_{p}}$ need not be de Rham).

When $g$ and $\underline{h}$ are cuspidal Hida families, the "weight two" classes $\kappa\left(f, g_{2}, h_{2}\right)$ attached to weight two specialisations $g_{2}$ and $h_{2}$ of $\underline{g}$ and $\underline{h}$ are obtained from the $p$-adic étale Abel-Jacobi image of a Gross-Kudla-Schoen diagonal cycles in the Chow group of nullhomologous codimension two cycles in the triple product of the modular curve $X_{1}\left(N p^{s}\right)$. It is worth noting that when passing from $k=1$ to $k>1$, the local root number at $\infty$ attached to $L\left(V_{f_{k} h_{k}}, s\right)$ changes sign (while the other root numbers stay the same), so that this $L$-function vanishes to odd order at its centre. The presence of Gross-KudlaSchoen diagonal cycles in this range is consistent with the Beilinson-Bloch conjecture for $L\left(V_{f g_{k} h_{k}}, s\right)$ and in fact provides evidence for it. (Cf. the preprint [38] of Yuan-ZhangZhang, where the case $k=2$ is studied.) The fact that the extension $\kappa\left(f, g_{\alpha}, h_{\alpha}\right)$ does not arise directly in $p$-adic étale cohomology, but only as a $p$-adic limit of geometric Galois representations, explains why $\kappa\left(f, g_{\alpha}, h_{\alpha}\right)$ need not be cristalline at $p$ in general.

The analogy with the work of Kato $[23,32]$ arises when the cuspidal Hida families $\underline{g}$ and $\underline{h}$ are replaced by Hida families of Eisenstein series. A global class $\kappa_{B K}\left(f, g_{\alpha}, h_{\alpha}\right)$, designated as the Beilinson-Kato class attached to $\left(f, g_{\alpha}, h_{\alpha}\right)$, is then defined as in (15), but replacing the étale Abel-Jacobi images $\kappa\left(f, g_{2}, h_{2}\right)$ by $p$-adic étale regulators of Beilinson elements in the higher Chow group $K_{2}\left(X_{1}\left(N p^{s}\right)\right)=\mathrm{CH}^{2}\left(X_{1}\left(N p^{s}\right)\right.$, 2) attached to a pair of modular units whose logarithmic derivatives give rise to $g_{2}$ and $h_{2}$. We refer the reader to [6] for more details in this setting.

In the intermediate setting where exactly one of $g$ and $\underline{h}$ (say, $g$ ) is cuspidal (and thus $\underline{h}$ is Eisenstein), global classes $\kappa\left(f, g_{2}, h_{2}\right)$ can be constructed geometrically as $p$-adic étale 
regulators of suitable Beilinson-Flach elements in the higher Chow group $K_{1}\left(X_{1}\left(N p^{s}\right)^{2}\right)=$ $\mathrm{CH}^{2}\left(X_{1}\left(N p^{s}\right)^{2}, 1\right)$ attached to a modular unit whose logarithmic derivative is $h_{2}$. The limit cohomology class arising in (15) is then denoted $\kappa_{B F}\left(f, g_{\alpha}, h_{\alpha}\right)$ and called the BeilinsonFlach class attached to the triple $\left(f, g_{\alpha}, h_{\alpha}\right)$. The Beilinson-Flach classes in $p$-adic families were introduced and studied in [2,10] and [11]. See also [27] and [24] for more recent work leading to substantial extensions and refinements of the results of loc.cit. in the setting of Beilinson-Flach elements.

The $p$-adic Selmer group

$$
H_{\text {fin }}^{1}\left(\mathbb{Q}, V_{f g h}\right):=\operatorname{Ext}_{\text {cris }}^{1}\left(\mathbb{Q}_{p}, V_{f g h}\right)
$$

attached to $E$ and $V_{g h}$ are the group of extensions of $\mathbb{Q}_{p}$ by $V_{f g h}$ in the category of $\mathbb{Q}_{p}$-linear representations of $G_{\mathbb{Q}}$ that are cristalline at $p$. Let

$$
\left(E(H)_{L} \otimes V_{12}\right)^{\operatorname{Gal}(H / \mathbb{Q})}, \quad \text { where } E(H)_{L}:=E(H) \otimes_{\mathbb{Z}} L
$$

denote the $\varrho_{12}$-isotypic part of the Mordell-Weil group of $E$. It is a finite-dimensional $L$-vector space by the Mordell-Weil theorem and is equipped with a natural inclusion

$$
\left(E(H)_{L} \otimes V_{12}\right)^{\operatorname{Gal}(H / \mathbb{Q})} \subset H_{\text {fin }}^{1}\left(H, V_{p}(E) \otimes_{\mathbb{Q}_{p}} V_{g h}\right)^{\operatorname{Gal}(H / \mathbb{Q})}=H_{\text {fin }}^{1}\left(\mathbb{Q}, V_{f g h}\right)
$$

induced from the connecting homomorphism $\delta$ of Kummer theory for $E(H)$ and the map $j_{g h}$ of (13).

When $L\left(E, \varrho_{g h}, s\right)$ has a double zero at $s=1$, Conjecture $\operatorname{BSD}\left(E, \varrho_{g h}\right)$ described in the previous section predicts that the associated Mordell-Weil and Selmer group in (18) is two-dimensional over $L$ and $\mathbb{Q}_{p}$, respectively. The finiteness of the relevant ShafarevichTate group furnishes the Selmer group with a natural $L$-rational structure

$$
\operatorname{Hom}_{G_{\mathbb{Q}}}\left(V_{12}, E(H)_{L}\right) \subset H_{\text {Sel }}^{1}\left(\mathbb{Q}, V_{f g h}\right) .
$$

As mentioned above, the vanishing of the central critical value $L\left(E, \varrho_{g h}, 1\right)$ implies that the generalised Kato classes in (14) are cristalline at $p$ and thus belong to $H_{\text {fin }}^{1}\left(\mathbb{Q}, V_{f g h}\right)$. The main goal of this article is to give a precise conjectural description of the position of the generalised Kato classes in $H_{\text {fin }}^{1}\left(\mathbb{Q}, V_{f g h}\right)$ relative to the $L$-structure given by (18), in a way that recovers older conjectures of Kato and Perrin-Riou in the setting of BeilinsonKato classes when $g$ and $h$ are Eisenstein series, and is consistent with the theorems and conjectures of [19] and [15].

\subsection{Basic properties}

In this section we recall some of the main properties of the generalised Kato classes already established in [19] and [15].

Restricting (17) to $G_{\mathbb{Q}_{p}}$, let

$$
H_{\text {fin }}^{1}\left(\mathbb{Q}_{p}, V_{f g h}\right):=\operatorname{Ext}_{\text {fin, } \mathbb{Q}_{p}}^{1}\left(\mathbb{Q}_{p}, V_{f g h}\right)
$$

denote the group of cristalline extensions of $\mathbb{Q}_{p}$ by $V_{f g h}$ in the category of $\mathbb{Q}_{p}$-linear representations of $G_{\mathbb{Q}_{p}}$, and let $H_{\text {sing }}^{1}\left(\mathbb{Q}_{p}, V_{f g h}\right):=H^{1}\left(\mathbb{Q}_{p}, V_{f g h}\right) / H_{\text {fin }}^{1}\left(\mathbb{Q}_{p}, V_{f g h}\right)$ denote the "singular quotient" of the local cohomology at $p$. Recall that $L$ has been chosen to be large enough to contain the frobenius eigenvalues $\alpha_{g}, \beta_{g}, \alpha_{h}$, and $\beta_{h}$, which therefore belong to $\mathbb{Q}_{p}$. Let 


$$
V_{g}^{\alpha}, V_{g}^{\beta} \subset V_{g}, \quad V_{h}^{\alpha}, V_{h}^{\beta} \subset V_{h}
$$

be the eigenspaces in $V_{g}$ and $V_{h}$, respectively, associated to these eigenvalues, and set

$$
\begin{aligned}
V_{g h}^{\alpha \alpha}:=V_{g}^{\alpha} \otimes V_{h}^{\alpha}, \quad V_{g h}^{\alpha \beta}:=V_{g}^{\alpha} \otimes V_{h}^{\beta}, \quad V_{g h}^{\beta \alpha}:=V_{g}^{\beta} \otimes V_{h}^{\alpha}, \\
V_{g h}^{\beta \beta}:=V_{g}^{\beta} \otimes V_{h}^{\beta}
\end{aligned}
$$

of $V_{g h}$. Even though $V_{g}$ and $V_{h}$ are both assumed to be regular at $p$, the same need not be true for $V_{g h}$, and in this case

$$
V_{g h}=V_{g h}^{\alpha \alpha} \oplus V_{g h}^{\alpha \beta} \oplus V_{g h}^{\beta \alpha} \oplus V_{g h}^{\beta \beta}
$$

gives a strict refinement of the decomposition of $V_{g h}$ into frobenius eigenspaces. In fact, some of the most interesting arithmetic applications of the generalised Kato classes (notably those spelled out in Sects. 4.3, 4.4, and 4.5) arise when $V_{g h}$ is not regular at $p$.

The first basic result extends Kato's explicit reciprocity law (corresponding to the case where $g$ and $h$ are both Eisenstein series) to the setting where both $g$ and $h$ are cuspidal (Theorem C of [19]) as well as to the intermediate Beilinson-Flach setting (Theorem 3.10 of [11]).

Theorem 3.1 The natural image of $\kappa\left(f, g_{\alpha}, h_{\alpha}\right)$ in $H_{\text {sing }}^{1}\left(\mathbb{Q}_{p}, V_{f g h}\right)$ belongs to $H_{\text {sing }}^{1}\left(\mathbb{Q}_{p}, V_{f} \otimes\right.$ $\left.V_{g h}^{\beta \beta}\right)$, and analogously for the remaining classes of (14). Moreover, the following are equivalent:

(1) For all choices of $\pi$ in (16), the generalised Kato classes of (14) belong to the BlochKato Selmer group of $V_{f g h}$, i.e. their images in $H_{\text {sing }}^{1}\left(\mathbb{Q}_{p}, V_{f g h}\right)$ are trivial;

(2) the central critical value $L\left(E, V_{g h}, 1\right)$ vanishes.

Assume from now on that $L\left(E, V_{g h}, 1\right)=0$, so that

(1) the $L$-series $L\left(E, V_{g h}, s\right)$ has a zero of even order $\geq 2$ at $s=1$;

(2) the generalised Kato classes of (14) belong to the Selmer group attached to $E$ and $V_{g h}$.

One is then naturally interested in a formulating non-vanishing criterion for these Selmer classes:

Conjecture 3.2 The generalised Kato classes in (14) generate a non-trivial subgroup of the Selmer group of $V_{\text {fgh }}$ for a suitable choice of $\pi$ in (16), if and only if the following equivalent conditions are satisfied:

(a) The L-series $L\left(E, V_{g h}, s\right)$ has a double zero at $s=1$;

(b) the Mordell-Weil group $\left(E(H)_{L} \otimes V_{12}\right)^{G Q}$ is two-dimensional over L;

(c) the Selmer group $H_{\text {fin }}^{1}\left(\mathbb{Q}, V_{f g h}\right)$ is two-dimensional over $\mathbb{Q}_{p}$.

Remark 3.3 Although the equivalence of conditions (a), (b), and (c) certainly lies very deep, it is part of a well-established conjecture, namely $\operatorname{BSD}\left(E, V_{g h}\right)$. The main novelty of Conjecture 3.2 is in providing a criterion for the non-triviality of the space generated by the generalised Kato classes. Note that Conjecture 3.2 does not predict that all four of the classes in (14) are non-trivial, nor even that these four classes generate the Selmer group, when (a), (b), and/or (c) are satisfied. These stronger conclusions are expected to be false in general, as illustrated by some of the examples in Sect. 4. 
Let

$$
\kappa_{p}\left(f, g_{\alpha}, h_{\alpha}\right)=\operatorname{res}_{p}\left(\kappa\left(f, g_{\alpha}, h_{\alpha}\right)\right)
$$

denote the image of the global class $\kappa\left(f, g_{\alpha}, h_{\alpha}\right)$ in the local cohomology group

$$
H_{\text {fin }}^{1}\left(\mathbb{Q}_{p}, V_{f g h}\right)=\left(H_{\text {fin }}^{1}\left(H_{p}, V_{f}\right) \otimes V_{g h}\right)^{\operatorname{Gal}\left(H_{p} / \mathbb{Q}_{p}\right)}=\left(E\left(H_{p}\right) \otimes V_{g h}\right)^{\operatorname{Gal}\left(H_{p} / \mathbb{Q}_{p}\right)} .
$$

As we describe more explicitly below, Theorem D of [19] asserts that this image is controlled by suitable $p$-adic avatars of the second derivative of the classical $L$-series $L\left(f, V_{g h}, s\right)$ at the central critical point $s=1$.

These $p$-adic values were defined and explored in [19] and [15] and are denoted

$$
\mathscr{L}_{p}^{g_{\alpha}}\left(\breve{f}, \breve{g}^{*}, \breve{h}\right), \quad \mathscr{L}_{p}^{g_{\beta}}\left(\breve{f}, \breve{g}^{*}, \breve{h}\right), \quad \mathscr{L}_{p}^{h_{\alpha}}\left(\breve{f}, \breve{g}, \breve{h}^{*}\right), \quad \mathscr{L}_{p}^{h_{\beta}}\left(\breve{f}, \breve{g}, \breve{h}^{*}\right) .
$$

They depend on the choice of certain test vectors

$$
(\breve{f}, \breve{g}, \breve{h}) \in S_{2}(N ; L) \times M_{1}(N, \chi ; L) \times M_{1}\left(N, \chi^{-1} ; L\right)
$$

with the same system of Hecke eigenvalues as $f, g$, and $h$, respectively, and with fourier coefficients in $L$, and on the choice of dual test vectors

$$
\left(\breve{g}^{*}, \breve{h}^{*}\right) \in \operatorname{Hom}\left(M_{1}\left(N, \chi^{-1} ; L\right), L\right) \times \operatorname{Hom}\left(M_{1}(N, \chi ; L), L\right)
$$

with the same system of Hecke eigenvalues as $g$ and $h$. We refer to the introduction of [19] for more details on their definition, contenting ourselves with remark that the $p$-adic $L$-value $\mathscr{L}_{p}{ }^{g_{\alpha}}\left(\breve{f}, \breve{g}^{*}, \breve{h}\right)$ is defined essentially as the $p$-adic limit of central critical values

$$
\mathscr{L}_{p}{ }^{g_{\alpha}}\left(\breve{f}, \breve{g}^{*}, \breve{h}\right):=\lim _{\ell \rightarrow 1} \mathcal{E}\left(f, g_{\ell}, h\right) \times C\left(\check{f}, \breve{g}^{*}, \breve{h}\right) \times \frac{L\left(V_{f} \otimes V_{g_{\ell}} \otimes V_{h}(\ell+1) / 2\right)}{\left\langle g_{\ell}, g_{\ell}\right\rangle},
$$

as $g_{\ell}$ ranges over the specialisations of (odd) weight $\ell \geq 3$ of the Hida family $g$ specialising to $g_{\alpha}$ in weight one. Here $\mathcal{E}\left(f, g_{\ell}, h\right)$ is a $p$-adic multiplier arising from a recipe of Panciskin, whose presence allows the $p$-adic interpolation of the special values above, and $C\left(\breve{f}, \breve{g}^{*}, \breve{h}\right)$ is a product over the primes dividing $N \cdot \infty$ of local terms which depend in a simple way on the choice of test vectors.

Choose a basis of $V_{g h}$ (over $\mathbb{Q}_{p}$, for now) which is compatible with the decomposition (21), i.e. choose nonzero vectors

$$
v_{g h}^{\alpha \alpha} \in V_{g h}^{\alpha \alpha}, \quad v_{g h}^{\alpha \beta} \in V_{g h}^{\alpha \beta}, \quad v_{g h}^{\beta \alpha} \in V_{g h}^{\beta \alpha}, \quad v_{g h}^{\beta \beta} \in V_{g h}^{\beta \beta} .
$$

Write

$$
\kappa_{p}\left(f, g_{\alpha}, h_{\alpha}\right)=R_{\alpha \alpha} \otimes v_{g h}^{\beta \beta}+R_{\alpha \beta} \otimes v_{g h}^{\beta \alpha}+R_{\beta \alpha} \otimes v_{g h}^{\alpha \beta}+R_{\beta \beta} \otimes v_{g h}^{\alpha \alpha} .
$$

The coordinate $R_{\xi}$ belongs to $E\left(H_{p}\right)_{\mathbb{Q}_{p}}^{\mathrm{Fr}_{p}=\xi}$, where $\xi$ ranges over the index set

$$
\left\{\alpha \alpha=\alpha_{g} \alpha_{h}, \quad \alpha \beta=\alpha_{g} \beta_{h}, \quad \beta \alpha=\beta_{g} \alpha_{h}, \quad \beta \beta=\beta_{g} \beta_{h}\right\} .
$$

Note that $R_{\xi}$ is even the image of a global point in $E(H)_{\mathbb{Q}_{p}}$, assuming the finiteness of the Shafarevich-Tate group of $E$ over $H$. Let

$$
\log _{p}: E\left(H_{p}\right)_{\mathbb{Q}_{p}} \longrightarrow H_{p}
$$

denote the formal group logarithm attached to an invariant differential on $E / \mathbb{Q}$. The following theorem is stated in Section 6.4 of [19]: 
Theorem 3.4 When $L\left(E, V_{g h}, 1\right)=0$, there exists a choice of $\pi$ in (16) and of test vectors for $f, g$, and $h$ such that the coordinates in (24) satisfy

$$
\log _{p}\left(R_{\alpha \beta}\right) \sim \mathscr{L}_{p}^{g_{\alpha}}\left(\breve{f}, \breve{g}^{*}, \breve{h}\right), \quad \log _{p}\left(R_{\beta \alpha}\right) \sim \mathscr{L}_{p}^{h_{\alpha}}\left(\breve{f}, \breve{g}, \breve{h}^{*}\right), \quad \log _{p}\left(R_{\beta \beta}\right)=0,
$$

where $\sim$ denotes equality up to a nonzero $p$-adic period in $H_{p}^{\times}$.

Remark 3.5 This theorem says nothing about the quantity $\log _{p}\left(R_{\alpha \alpha}\right)$, which does not bear any direct relationship with $p$-adic $L$-values introduced above. We expect that $\log _{p}\left(R_{\alpha \alpha}\right)$ may rather be connected with the first derivative of a putative refinement of $\mathscr{L}_{p}^{f}\left(f, g_{\alpha}, h_{\alpha}\right)$ in which all three modular forms would be made to vary in a Hida family.

As explained in the introduction and in Section 6.3. of [19], Theorem 3.4 has the following corollary which can be viewed as a $p$-adic Gross-Zagier formula in "analytic rank two":

Corollary 3.6 If $L\left(E, V_{g h}, 1\right)=0$ and $\mathscr{L}_{p}{ }^{g_{\alpha}}\left(\breve{f}, \breve{g}^{*}, \breve{h}\right) \neq 0$ for a suitable choice $\left(\breve{f}, \breve{g}^{*}, \breve{h}\right)$ of test vectors, then the two global classes

$$
\kappa\left(f, g_{\alpha}, h_{\alpha}\right), \quad \kappa\left(f, g_{\alpha}, h_{\beta}\right)
$$

are linearly independent in the Selmer group $H_{\text {fin }}^{1}\left(\mathbb{Q}, V_{f g h}\right)$ attached to $E$ and $V_{g h}$, for a suitable choice of $\pi$ in (16).

Theorem 3.4 and its corollary motivated the experimental study undertaken in [15] of the special values of $p$-adic $L$-functions appearing in (26). This led to a precise conjecture for these values up to a factor of $L^{\times}$rather than $\mathbb{Q}_{p}^{\times}$.

To formulate this conjecture, recall that the class $\kappa\left(f, g_{\alpha}, h_{\alpha}\right)$ is expected to be trivial when $\operatorname{ord}_{s=1} L\left(E, V_{g h}, s\right)>2$. Assume that this $L$-function has a double zero at the centre, which implies, by Conjecture $\operatorname{BSD}\left(E, V_{g h}\right)$, that $\left(E(H)_{L} \otimes V_{12}\right)^{G_{\mathbb{Q}}}$ is a two-dimensional $L$-vector space.

Fix vectors $v_{g h}^{\alpha \alpha}, \ldots, v_{g h}^{\beta \beta}$ chosen as in (23), with the difference that they belong to $L$ vector space $V_{12}$ rather than the $\mathbb{Q}_{p}$-vector space $V_{g h}$. Choose a basis $(P, Q)$ for this $L$-vector space, and write

$$
\begin{aligned}
& P=P_{\alpha \alpha} \otimes v_{g h}^{\beta \beta}+P_{\alpha \beta} \otimes v_{g h}^{\beta \alpha}+P_{\beta \alpha} \otimes v_{g h}^{\alpha \beta}+P_{\beta \beta} \otimes v_{g h}^{\alpha \alpha}, \\
& Q=Q_{\alpha \alpha} \otimes v_{g h}^{\beta \beta}+Q_{\alpha \beta} \otimes v_{g h}^{\beta \alpha}+Q_{\beta \alpha} \otimes v_{g h}^{\alpha \beta}+Q_{\beta \beta} \otimes v_{g h}^{\alpha \alpha},
\end{aligned}
$$

where $P_{\xi}, Q_{\xi}$ are points in $E(H)_{L}^{\mathrm{Fr}_{p}=\xi}$ for every $\xi \in\left\{\alpha \alpha=\alpha_{g} \alpha_{h}, \alpha \beta=\alpha_{g} \beta_{h}, \beta \alpha=\right.$ $\left.\beta_{g} \alpha_{h}, \beta \beta=\beta_{g} \beta_{h}\right\}$.

These points can be used to define a regulator attached to $g_{\alpha}$, whose entries are the $p$-adic formal group logarithms of the coordinates attached to the vectors $v_{g h}^{\alpha \alpha}$ and $v_{g h}^{\alpha \beta}$ (and similarly for $h_{\alpha}$ ):

Definition 3.7 The regulators attached to $E$ and $V_{12}$ are

$$
\begin{aligned}
\operatorname{Reg}_{g_{\alpha}}\left(E, V_{12}\right) & =\operatorname{det}\left(\begin{array}{ll}
\log _{p} P_{\beta \beta} & \log _{p} P_{\beta \alpha} \\
\log _{p} Q_{\beta \beta} & \log _{p} Q_{\beta \alpha}
\end{array}\right) \\
& =\log _{p} P_{\beta \beta} \cdot \log _{p} Q_{\beta \alpha}-\log _{p} Q_{\beta \beta} \cdot \log _{p} P_{\beta \alpha}, \\
\operatorname{Reg}_{h_{\alpha}}\left(E, V_{12}\right) & =\operatorname{det}\left(\begin{array}{ll}
\log _{p} P_{\beta \beta} & \log _{p} P_{\alpha \beta} \\
\log _{p} Q_{\beta \beta} & \log _{p} Q_{\alpha \beta}
\end{array}\right) \\
& =\log _{p} P_{\beta \beta} \cdot \log _{p} Q_{\alpha \beta}-\log _{p} Q_{\beta \beta} \cdot \log _{p} P_{\alpha \beta} .
\end{aligned}
$$


The main conjecture of [15] is the following, ${ }^{3}$ assuming $\frac{\alpha_{g}}{\beta_{g}} \neq \pm 1$ (resp. $\frac{\alpha_{h}}{\beta_{h}} \neq \pm 1$ ) so that the Stark unit $u_{g_{\alpha}}$ (resp. $u_{h_{\alpha}}$ ) is well defined:

Conjecture 3.8 Assume that $L\left(E, V_{g h}, s\right)$ vanishes to order 2 at $s=1$. Then there exists a choice of test vectors $\left(\breve{f}, \breve{g}^{*}, \breve{h}\right)$ and $\left(\breve{f}, \breve{g}, \breve{h}^{*}\right)$ such that

$$
\mathscr{L}_{p}^{g_{\alpha}}\left(\breve{f}, \breve{g}^{*}, \breve{h}\right)=\frac{\operatorname{Reg}_{g_{\alpha}}\left(E, V_{12}\right)}{\log _{p} u_{g_{\alpha}}}, \quad \mathscr{L}_{p}^{h_{\alpha}}\left(\breve{f}, \breve{g}, \breve{h}^{*}\right)=\frac{\operatorname{Reg}_{h_{\alpha}}\left(E, V_{12}\right)}{\log _{p} u_{h_{\alpha}}} \quad\left(\bmod L^{\times}\right) .
$$

Remark 3.9 Conjecture 3.8 lends itself to numerical verification and has been extensively tested in [15]. This is because the $p$-adic $L$-values $\mathscr{L}_{p}{ }^{g_{\alpha}}\left(\breve{f}, \breve{g}^{*}, \breve{h}\right)$ and $\mathscr{L}_{p}{ }^{h_{\alpha}}\left(\breve{f}, \breve{g}, \breve{h}^{*}\right)$ can be expressed in terms of the rather concrete $p$-adic iterated integrals of loc.cit., which can be computed efficiently using Alan Lauder's [26] fast ordinary projection algorithms on the space of overconvergent modular forms. In contrast, the generalised Kato classes themselves (like many objects constructed in étale cohomology) seem difficult to compute in practice, even though their theoretical usefulness is amply illustrated in [11] and [19].

\subsection{Enhanced regulators}

The goal of this article is to combine the insights arising from Theorem 3.4 and Conjecture 3.8 to formulate a conjecture on the position of the generalised Kato classes themselves in $\left(E(H) \otimes V_{g h}\right)^{G_{\mathbb{Q}}}$, specifying this position up to an ambiguity of $L^{\times}$rather than the less precise $\mathbb{Q}_{p}^{\times}$ambiguity of Theorem 3.4.

The most important ingredients in the formulation of this conjecture are the so-called enhanced regulators

$$
\begin{aligned}
& \widetilde{\operatorname{Reg}}\left(E, V_{12}\right) \in\left(E(H)_{L} \otimes V_{12}\right)^{G_{\mathbb{Q}}} \otimes\left(E(H)_{L} \otimes V_{12}\right)^{G \mathbb{Q},} \\
& \widetilde{\operatorname{Reg}}_{\alpha \alpha}\left(E, V_{12}\right) \in\left(H_{p}\right)^{\mathrm{Fr}_{p}=\beta_{g} \beta_{h} \otimes\left(E(H)_{L} \otimes V_{12}\right)^{G},} \\
& \widetilde{\operatorname{Reg}}\left(E, V_{g h}\right) \in\left(E(H)_{L} \otimes V_{g h}\right)^{G_{\mathbb{Q}}} \otimes\left(E(H)_{L} \otimes V_{g h}\right)^{G_{\mathbb{Q}},} \\
& \widetilde{\operatorname{Reg}}_{\alpha \alpha}\left(E, V_{g h}\right) \in D\left(V_{g h}^{\alpha \alpha}\right) \otimes\left(E(H)_{L} \otimes V_{g h}\right)^{G_{\mathbb{Q}},}
\end{aligned}
$$

whose definition is somewhat in the spirit of the regulator $R_{S}$ defined in equation (2) of [13], and which we now proceed to describe. As in (6), here $D\left(V_{g h}^{\alpha \alpha}\right):=\left(\mathbb{Q}_{p}^{\mathrm{nr}} \otimes V_{g h}^{\alpha \alpha}\right)^{G_{\mathbb{Q}_{p}}}=$ $\left(H_{p} \otimes V_{g h}^{\alpha \alpha}\right)^{G_{\mathbb{Q} p}}$.

Definition 3.10 Choose an $L$-basis $(P, Q)$ of the two-dimensional vector space $(E(H) \otimes$ $\left.V_{12}\right)^{G_{\mathbb{Q}}}$, and set

$$
\widetilde{\operatorname{Reg}}\left(E, V_{12}\right):=\operatorname{det}\left(\begin{array}{cc}
P & P \\
Q & Q
\end{array}\right):=P \otimes Q-Q \otimes P .
$$

It does not depend on the choice of basis that was made to define it, up to multiplication by $L^{\times}$.

The function $\log _{\alpha \alpha}:\left(E(H)_{L} \otimes V_{12}\right)^{G_{\mathbb{Q}}} \longrightarrow\left(H_{p}\right)^{\mathrm{Fr}_{p}=\beta_{g} \beta_{h}}$ defined by

$$
\log _{\alpha \alpha}(P):=\log _{p}\left(P_{\beta \beta}\right)
$$

${ }^{3}$ We warn the reader that here in this note we have chosen to state the main conjecture of [15] in terms of the arithmetic frobenius $\mathrm{Fr}_{p}$ at $p$, while in [15] we rather employ the geometric frobenius $\sigma_{p}=\mathrm{Fr}_{p}^{-1}$. It is for this reason that the roles of $\alpha$ and $\beta$ are swapped in both formulations. 
induces a linear map

$$
\begin{gathered}
\log _{\alpha \alpha} \otimes 1:\left(E(H)_{L} \otimes V_{12}\right)^{G_{\mathbb{Q}}} \otimes\left(E(H)_{L} \otimes V_{12}\right)^{G_{\mathbb{Q}}} \\
\longrightarrow\left(H_{p}\right)^{\mathrm{Fr}_{p}=\beta_{g} \beta_{h}} \otimes\left(E(H)_{L} \otimes V_{12}\right)^{G_{\mathbb{Q}}},
\end{gathered}
$$

and we set

$$
\widetilde{\operatorname{Reg}}_{\alpha \alpha}\left(E, V_{12}\right):=\left(\log _{\alpha \alpha} \otimes 1\right)\left(\widetilde{\operatorname{Reg}}\left(E, V_{12}\right)\right)=\log _{p}\left(P_{\beta \beta}\right) \otimes Q-\log _{p}\left(Q_{\beta \beta}\right) \otimes P .
$$

Recall the embedding $j_{g h}: V_{12} \longrightarrow V_{g h}^{L} \subset V_{g h}$ of (12). Although this embedding is completely non-canonical and only defined up to scaling by $\mathbb{Q}_{p}^{\times}$, there is a canonical way of embedding $V_{12}^{\otimes 2}$ into $V_{g h}^{\otimes 2}$. This is done by exploiting the canonical dualities on $V_{g}$ and $V_{h}$ described in Sect. 2, which gives rise to perfect pairings

$$
V_{g} \times V_{g} \longrightarrow \mathbb{Q}_{p}(\chi), \quad V_{h} \times V_{h} \longrightarrow \mathbb{Q}_{p}\left(\chi^{-1}\right), \quad V_{g h} \times V_{g h} \longrightarrow \mathbb{Q}_{p} .
$$

These pairings allow us to define $L$-rational structures $V_{g}^{L *}, V_{h}^{L *}$ and $V_{g h}^{L *}$ which are dual to $V_{g}^{L}, V_{h}^{L}$ and $V_{g h}^{L}$, respectively, by letting $V_{g}^{L *}$ be the $L$-dual of $V_{g}^{L}$ in $V_{g}$, and likewise for $V_{h}^{L *}$ and $V_{g h}^{L *}$. We may then choose $G_{\mathbb{Q}}$-equivariant embeddings

$$
j_{g}^{*}: V_{1} \longrightarrow V_{g}^{L *}, \quad j_{h}^{*}: V_{2} \longrightarrow V_{h}^{L *}, \quad j_{g h}^{*}:=j_{g}^{*} \otimes j_{h}^{*}: V_{12} \longrightarrow V_{g h}^{L *},
$$

which are well defined up to scaling by $L^{\times}$. Replacing $j_{g h}$ by $\mu \cdot j_{g h}$, for any $\mu \in \mathbb{Q}_{p}^{\times}$, has the effect of replacing $j_{g h}^{*}$ by $\mu^{-1} \cdot j_{g h}^{*}$. Hence, the map

$$
j_{g h} \otimes j_{g h}^{*}: V_{12} \otimes V_{12} \longrightarrow V_{g h} \otimes V_{g h}
$$

is well defined up to scaling by $L^{\times}$.

Definition 3.11 The enhanced regulator $\widetilde{\operatorname{Reg}}\left(E, V_{g h}\right)$ associated to $E$ and $V_{g h}$ is

$$
\widetilde{\operatorname{Reg}}\left(E, V_{g h}\right):=\left(j_{g h} \otimes j_{g h}^{*}\right)\left(\widetilde{\operatorname{Reg}}\left(E, V_{12}\right)\right) \in\left(E(H) \otimes V_{g h}\right)^{G_{\mathbb{Q}}} \otimes\left(E(H) \otimes V_{g h}\right)^{G_{\mathbb{Q}}} .
$$

Finally, let

$$
\log _{p}:\left(E(H) \otimes V_{g h}\right)^{G_{\mathbb{Q}}} \longrightarrow\left(H_{p} \otimes V_{g h}\right)^{G_{\mathbb{Q}}}=D\left(V_{g h}\right)
$$

be the canonical $p$-adic logarithm map induced from the $p$-adic logarithm of (25) via the fixed embedding $H \subset H_{p}$, and let

$$
\log _{\alpha \alpha}:\left(E(H) \otimes V_{g h}\right)^{G_{\mathbb{Q}}} \longrightarrow D\left(V_{g h}^{\alpha \alpha}\right)
$$

be its composition with the functorial projection $D\left(V_{g h}\right) \longrightarrow D\left(V_{g h}^{\alpha \alpha}\right)$. This logarithm map is just the more canonical counterpart of the map $\log _{\alpha \alpha}$ : the latter depends on the choice of a basis vector $v_{g h}^{\alpha \alpha}$ for $V^{\alpha \alpha}$ and is related to $\log _{\alpha \alpha}$ by the rule

$$
\log _{\alpha \alpha}:=\log _{\alpha \alpha} \otimes v_{g h}^{\alpha \alpha} .
$$

We set

$$
\widetilde{\operatorname{Reg}}_{\alpha \alpha}\left(E, V_{g h}\right):=\left(\log _{\alpha \alpha} \otimes 1\right)\left(\widetilde{\operatorname{Reg}}\left(E, V_{g h}\right)\right)=\log _{\alpha \alpha}(P) \otimes Q-\log _{\alpha \alpha}(Q) \otimes P .
$$

It is worth noting that the enhanced regulator $\widetilde{\operatorname{Reg}}_{\alpha \alpha}\left(E, V_{g h}\right)$ is a canonical invariant associated to $E$ and $V_{g h}$, i.e. it is well defined up to multiplication by $L^{\times}$, while the less canonical $\widetilde{\operatorname{Reg}}_{\alpha \alpha}\left(E, V_{12}\right)$ depends on the choice of a basis $v_{g h}^{\alpha \alpha}$ for $V_{g h}^{\alpha \alpha}$. The two regulators are related by

$$
\widetilde{\operatorname{Reg}}_{\alpha \alpha}\left(E, V_{g h}\right)=\widetilde{\operatorname{Reg}}_{\alpha \alpha}\left(E, V_{12}\right) \otimes v_{g h}^{\alpha \alpha} .
$$




\subsection{The conjecture}

Recall the periods

$$
\omega_{g_{\alpha}} \in D\left(V_{g}^{\alpha}\right), \quad \omega_{h_{\alpha}} \in D\left(V_{h}^{\alpha}\right)
$$

constructed in (6). The main conjecture of this note is:

Conjecture 3.12 Assume that $r\left(E, V_{g h}\right)=2$. The generalised Kato class $\kappa\left(f, g_{\alpha}, h_{\alpha}\right)$ belongs to $\left(E(H) \otimes V_{g h}\right)_{\mathbb{Q}}$ and satisfies the relation

$$
\omega_{g_{\alpha}} \omega_{h_{\alpha}} \otimes \kappa\left(f, g_{\alpha}, h_{\alpha}\right) \sim_{L} \widetilde{\operatorname{Reg}}_{\alpha \alpha}\left(E, V_{g h}\right)
$$

in $D\left(V_{g h}^{\alpha \alpha}\right) \otimes\left(E(H) \otimes V_{g h}\right)^{G_{\mathbb{Q}}}$, where $\sim_{L}$ denotes an equality up to scaling by a factor in $L$ which is nonzero for a suitable choice of $\pi$ in (16).

The following proposition shows that, under Conjecture 2.1 (relating the canonical period attached to $g$ to the Stark unit $u_{g_{\alpha}}$ ) and Conjecture 3.2 (a mild strengthening of $\left.\operatorname{BSD}\left(E, \varrho_{g h}\right)\right)$, Conjecture 3.12 implies the main conjecture of [15]. Before dismissing this proposition as mere conjectural relations between conjectures, the reader is reminded that Conjecture 3.8 lends itself to experiment and has been extensively tested numerically in [15], while the strengthening described in Conjecture 3.12 lies for the moment beyond the range of explicit calculations (cf. Remark 3.9).

Proposition 3.13 Assume Conjectures 2.1 and 3.2. Then Conjecture 3.12 implies Conjecture 3.8.

Proof Consider the product of periods

$$
\eta_{g_{\alpha}} \omega_{h_{\alpha}}=\left(\Xi_{g_{\alpha}} \otimes v_{g}^{\beta}\right) \cdot\left(\Omega_{h_{\alpha}} \otimes v_{h}^{\alpha}\right)=\Xi_{g_{\alpha}} \cdot \Omega_{h_{\alpha}} \otimes v_{g h}^{\beta \alpha} \in D\left(V_{g h}^{\beta \alpha}\right)
$$

defined in Sect. 2.

The pairing introduced in (7) gives rise to a pairing

$$
\langle,\rangle: D\left(V_{g h}^{\alpha \beta}\right) \times D\left(V_{g h}^{\beta \alpha}\right) \longrightarrow D\left(\mathbb{Q}_{p}\right)=\mathbb{Q}_{p} .
$$

As shown in the proof of [19, Theorem 6.10 (ii)],

$$
\left\langle\log _{\alpha \beta} \kappa\left(f, g_{\alpha}, h_{\alpha}\right), \eta_{g_{\alpha}} \omega_{h_{\alpha}}\right\rangle=\mathscr{L}_{p}^{g_{\alpha}}(f, g, h) \quad\left(\bmod L^{\times}\right) .
$$

On the other hand, by the definition of the enhanced regulator,

$$
\begin{aligned}
\log _{\alpha \beta} \widetilde{\operatorname{Reg}}_{\alpha \alpha}\left(E, V_{g h}\right) & =\left(\log _{p} P_{\beta \beta} \log _{p} Q_{\beta \alpha}-\log _{p} Q_{\beta \beta} \log _{p} P_{\beta \alpha}\right) \otimes v_{g h}^{\alpha \alpha} \otimes v_{g h}^{* \alpha \beta} \\
& =\operatorname{Reg}_{g_{\alpha}}\left(E, V_{12}\right) \otimes v_{g h}^{\alpha \alpha} \otimes v_{g h}^{* \alpha \beta}\left(\bmod L^{\times}\right) .
\end{aligned}
$$

Hence, the following equality holds in $D\left(V_{g h}^{\alpha \alpha}\right)$ :

$$
\left\langle\log _{\alpha \beta} \widetilde{\operatorname{Reg}}_{\alpha \alpha}\left(E, V_{g h}\right), \eta_{g_{\alpha}} \omega_{h_{\alpha}}\right\rangle=\Xi_{g_{\alpha}} \cdot \Omega_{h_{\alpha}} \cdot \operatorname{Reg}_{g_{\alpha}}\left(E, V_{g h}\right) \otimes v_{g h}^{\alpha \alpha} \quad\left(\bmod L^{\times}\right) .
$$

By pairing the value of $\log _{\alpha \beta}$ at both sides of the displayed identity in Conjecture 3.12 with the class $\eta_{g_{\alpha}} \omega_{h_{\alpha}}$ and invoking (32) and (33), we obtain

$$
\omega_{g_{\alpha}} \omega_{h_{\alpha}} \otimes \mathscr{L}_{p}^{g_{\alpha}}(f, g, h)=\Xi_{g_{\alpha}} \cdot \Omega_{h_{\alpha}} \cdot \operatorname{Reg}_{g_{\alpha}}\left(E, V_{12}\right) \otimes v_{g h}^{\alpha \alpha} \in D\left(V_{g h}^{\alpha \alpha}\right) \quad\left(\bmod L^{\times}\right) .
$$

Since

$$
\omega_{g_{\alpha}} \omega_{h_{\alpha}}=\Omega_{g_{\alpha}} \cdot \Omega_{h_{\alpha}} \cdot v_{g h}^{\alpha \alpha}\left(\bmod L^{\times}\right),
$$


it follows that

$$
\Omega_{g_{\alpha}} \mathscr{L}_{p}^{g_{\alpha}}(f, g, h)=\Xi_{g_{\alpha}} \operatorname{Reg}_{g_{\alpha}}\left(E, V_{12}\right) \quad\left(\bmod L^{\times}\right),
$$

and therefore that

$$
\mathscr{L}_{p}^{g_{\alpha}}(f, g, h)=\frac{\operatorname{Reg}_{g_{\alpha}}\left(E, V_{12}\right)}{\mathcal{L}_{g_{\alpha}}}\left(\bmod L^{\times}\right) .
$$

Conjecture 3.8 now follows directly from this equality after invoking Conjecture 2.1.

Remark 3.14 As explained in a number of the examples covered in Sect. 4 below, it may happen that all four of the $p$-adic iterated integrals in (22) are equal to zero even when some of the generalised Kato classes are non-trivial. This suggests that Conjecture 3.12 is a genuine strengthening of Conjecture 3.8.

\section{Special cases}

This section examines Conjecture 3.12, and the special forms taken by the enhanced regulators

$$
\widetilde{\operatorname{Reg}}_{\alpha \alpha}\left(E ; V_{12}\right), \quad \widetilde{\operatorname{Reg}}_{\alpha \beta}\left(E ; V_{12}\right), \quad \widetilde{\operatorname{Reg}}_{\beta \alpha}\left(E ; V_{12}\right), \quad \widetilde{\operatorname{Reg}}_{\beta \beta}\left(E ; V_{12}\right),
$$

in the arithmetically interesting cases where $V_{g h}$ is reducible. According to Darmon et al. $[16, \mathbb{\$}]$, the following is a complete list of scenarios where this occurs:

(1) The original Beilinson-Kato setting where $V_{g}$ and $V_{h}$ are both reducible, i.e. where $g$ and $h$ are both Eisenstein series of weight one;

(2) the Beilinson-Flach setting where exactly one of $V_{g}$ or $V_{h}$ is reducible, i.e. where exactly one of $g$ or $h$ is cuspidal;

(3) the complex multiplication case where $V_{g}$ and $V_{h}$ are both induced from characters of a common imaginary quadratic field;

(4) the real multiplication case where $V_{g}$ and $V_{h}$ are induced from characters of mixed signature of a common real quadratic field;

(5) the adjoint case where $h$ is (a twist of) the dual of $g$, so that $V_{g h}$ is the direct sum of a one-dimensional representation and a twist of the adjoint of $V_{g}$.

The reader will notice that some of the above settings arise when $\varrho_{g}$ and/or $\varrho_{h}$ are reducible, while in Sects. 2 and 3 these representations were assumed to be irreducible. This assumption was imposed to a large extent for the sake of simplicity of the exposition, and the statement (and presumed validity) of Conjecture 3.12 does not rely on it. For completeness, we have therefore described the enhanced regulators that appear in Conjecture 3.12 in all of the above cases.

\subsection{Beilinson-Kato classes}

Assume that $g$ and $h$ are both Eisenstein series. After possibly twisting $g$ or $h$, there is no real loss of generality in assuming that there exist Dirichlet characters $\chi_{1}, \chi_{2}$ such that $g$ and $h$ are given by

$$
g=E_{1}\left(\chi_{1}, \chi_{2}\right), \quad h=E_{1}\left(1, \chi_{12}^{-1}\right), \quad \text { where } \quad \chi_{12}=\chi_{1} \chi_{2} .
$$

We refer to e.g. [10, \$2.1.2] for the definition of these weight one Eisenstein series in terms of their $q$-expansions. The Galois representations attached to $g$ and $h$ are reducible, 
namely

$$
\begin{gathered}
V_{1}=L\left(\chi_{1}\right) \oplus L\left(\chi_{2}\right), \quad V_{2}=L \oplus L\left(\chi_{12}^{-1}\right), \\
V_{12}=L\left(\chi_{1}\right) \oplus L\left(\chi_{1}^{-1}\right) \oplus L\left(\chi_{2}\right) \oplus L\left(\chi_{2}^{-1}\right),
\end{gathered}
$$

where the coefficient field $L$ is the cyclotomic field generated by the images of $\chi_{1}$ and $\chi_{2}$. These representations factor through the Galois group $\mathrm{Gal}(H / \mathbb{Q})$ of an abelian extension $H$ of $\mathbb{Q}$. We may set

$$
\alpha_{g}=\chi_{1}(p), \quad \beta_{g}=\chi_{2}(p), \quad \alpha_{h}=1, \quad \beta_{h}=\chi_{12}^{-1}(p)
$$

The regularity assumption implies that $V_{1}$ and $V_{2}$ decompose uniquely as a direct sum of two $G_{\mathbb{Q}_{p}}$-stable lines, which are also stable under $G_{\mathbb{Q}}$. More precisely,

$$
V_{12}^{\alpha \alpha}=L \cdot v_{\chi_{1}}, \quad V_{12}^{\beta \beta}=L \cdot v_{\bar{\chi}_{1}}, \quad V_{12}^{\alpha \beta}=L \cdot v_{\bar{\chi}_{2}}, \quad V_{12}^{\beta \alpha}=L \cdot v_{\chi_{2}},
$$

where $\left(v_{\chi_{1}}, v_{\bar{\chi}_{1}}, v_{\bar{\chi}_{2}}, v_{\chi_{2}}\right)$ is a basis for $V_{12}$ on which $G_{\mathbb{Q}}$ acts via the characters $\chi_{1}, \bar{\chi}_{1}, \bar{\chi}_{2}$, and $\chi_{2}$, respectively.

The class $\kappa\left(f, g_{\alpha}, h_{\alpha}\right)=\kappa_{\mathrm{BK}}\left(f, g_{\alpha}, h_{\alpha}\right)$ was constructed by Kato as a $p$-adic limit of Beilinson elements attached to pairs of modular units whose logarithmic derivatives are weight two Eisenstein series. Theorem 3.1 in this case boils down to Kato's reciprocity law, which asserts that $\kappa\left(f, g_{\alpha}, h_{\alpha}\right)$ belongs to the Selmer group of $E$ over $H$ if and only if the $L$-function

$$
L\left(E, V_{g h}, s\right)=L\left(E, \chi_{1}, s\right) L\left(E, \bar{\chi}_{1}, s\right) L\left(E, \chi_{2}, s\right) L\left(E, \bar{\chi}_{2}, s\right)
$$

vanishes at $s=1$. In this case, it clearly vanishes to even order and vanishes to order two if and only if (after eventually interchanging the characters $\chi_{1}$ and $\chi_{2}$ )

$$
\operatorname{ord}_{s=1} L\left(E, \chi_{1}, s\right)=\operatorname{ord}_{s=1} L\left(E, \bar{\chi}_{1}, s\right)=1, \quad L\left(E, \chi_{2}, 1\right), L\left(E, \bar{\chi}_{2}, 1\right) \neq 0 \text {. }
$$

Assuming that this is the case, Conjectures $\operatorname{BSD}\left(E, \chi_{1}\right)$ and $\operatorname{BSD}\left(E, \chi_{2}\right)$ predict that $\left(E(H)_{L} \otimes V_{12}\right)^{G_{\mathbb{Q}}}$ is two-dimensional over $L$ and that a basis for it can be chosen to be

$$
P:=P_{\bar{\chi}_{1}} \otimes v_{\chi_{1}}, \quad Q:=Q_{\chi_{1}} \otimes v_{\bar{\chi}_{1}},
$$

where $P_{\bar{\chi}_{1}}$ and $Q_{\chi_{1}}$ are global points in $E(H)_{L}$ generating the $\bar{\chi}_{1}$ and $\chi_{1}$ eigenspaces, respectively, for the natural action of $G_{\mathbb{Q}}$. With these notations, we have

$$
\begin{aligned}
& P_{\alpha \alpha}=P_{\alpha \beta}=P_{\beta \alpha}=0, \quad P_{\beta \beta}=P_{\bar{\chi}_{1}}, \\
& Q_{\alpha \beta}=Q_{\beta \alpha}=Q_{\beta \beta}=0, \quad Q_{\alpha \alpha}=Q_{\chi_{1}} .
\end{aligned}
$$

This immediately implies that

$$
\begin{array}{ll}
\widetilde{\operatorname{Reg}}_{\alpha \alpha}\left(E, V_{12}\right)=\log _{p}\left(P_{\bar{\chi}_{1}}\right) \cdot Q, & \widetilde{\operatorname{Reg}}_{\alpha \beta}\left(E, V_{12}\right)=0, \\
\widetilde{\operatorname{Reg}}_{\beta \alpha}\left(E, V_{12}\right)=0, & \widetilde{\operatorname{Reg}}_{\beta \beta}\left(E ; V_{12}\right)=\log _{p}\left(Q_{\chi_{1}}\right) \cdot P .
\end{array}
$$

It follows that

$$
\operatorname{Reg}_{g_{\alpha}}\left(E ; V_{12}\right)=\operatorname{Reg}_{g_{\beta}}\left(E ; V_{12}\right)=\operatorname{Reg}_{h_{\alpha}}\left(E ; V_{12}\right)=\operatorname{Reg}_{h_{\beta}}\left(E ; V_{12}\right)=0 .
$$

This accounts for the fact that the $p$-adic iterated integrals

$$
\mathscr{L}_{p}^{g_{\alpha}}(f, g, h), \quad \mathscr{L}_{p}^{g_{\beta}}(f, g, h), \quad \mathscr{L}_{p}^{h_{\alpha}}(f, g, h), \quad \mathscr{L}_{p}^{h_{\beta}}(f, g, h)
$$


systematically vanish ${ }^{4}$ when $g$ and $h$ are Eisenstein series that are regular at $p$. Conjecture 3.12 makes the stronger prediction that the generalised Kato classes themselves are nontrivial, and is consistent with a Conjecture of Perrin-Riou, since it predicts that

$$
\log _{\beta \beta}\left(\kappa\left(f, g_{\alpha}, h_{\alpha}\right)\right)=\log _{\alpha \alpha}\left(\kappa\left(f, g_{\beta}, h_{\beta}\right)\right)=\log _{p}\left(P_{\bar{\chi}_{1}}\right) \log _{p}\left(Q_{\chi_{1}}\right) \quad\left(\bmod L^{\times}\right) .
$$

\subsection{Beilinson-Flach classes}

In the Beilinson-Flach setting, it can be assumed without loss of generality that $g$ is a weight one cusp form with nebentypus character $\chi$ and Galois representation $V_{g}=V_{1} \otimes_{L} \mathbb{Q}_{p}$, and that $h:=E_{1}\left(1, \chi^{-1}\right)$ is the weight one Eisenstein series attached to the pair $\left(1, \chi^{-1}\right)$ of Dirichlet characters. The relevant four-dimensional representations are then equal to

$$
V_{g h}=V_{g} \oplus V_{\bar{g}} ; \quad V_{12}=V_{1} \oplus \bar{V}_{1},
$$

and the Hasse-Weil-Artin $L$-series

$$
L\left(E, V_{g h}, s\right)=L\left(E, V_{g}, s\right) L\left(E, \bar{V}_{g}, s\right)
$$

has a double zero at $s=1$ precisely when each of the primitive $L$-series $L\left(E, V_{g}, s\right)$ and $L\left(E, V_{\bar{g}}, s\right)$ have a simple zero at $s=1$. Conjecture $\operatorname{BSD}\left(E, V_{g}\right)$ then implies that each of the $L$-vector spaces on the right-hand side of

$$
\left.\left(E(H)_{L} \otimes V_{12}\right)^{G_{\mathbb{Q}}}\right)=\left(E(H)_{L} \otimes V_{1}\right)^{G_{\mathbb{Q}}} \oplus\left(E(H)_{L} \otimes \bar{V}_{1}\right)^{G_{\mathbb{Q}}},
$$

is one-dimensional. Let $P$ be an $L$-basis for $\left(E(H)_{L} \otimes V_{1}\right)^{G_{\mathbb{Q}}}$ and let $\bar{P}$ be the associated $L$-basis for $\left(E(H)_{L} \otimes \bar{V}_{1}\right)^{G_{\mathbb{Q}}}$, obtained by applying complex conjugation to the coefficients in $L$.

After fixing an ordering $\alpha_{g}, \beta_{g} \in L$ for the eigenvalues of $\operatorname{Fr}_{p}$ on $V_{1}$, and setting

$$
\alpha_{h}=1, \quad \beta_{h}=\chi^{-1}(p)=\left(\alpha_{g} \beta_{g}\right)^{-1},
$$

we have

$$
V_{12}^{\alpha \alpha}=V_{1}^{\alpha_{g}}, \quad V_{12}^{\alpha \beta}=\bar{V}_{1}^{\beta_{g}^{-1}}, \quad V_{12}^{\beta \alpha}=V_{1}^{\beta_{g}}, \quad V_{12}^{\beta \beta}=\bar{V}_{1}^{\alpha_{g}^{-1}},
$$

and hence

$$
\begin{array}{llll}
P_{\alpha \alpha}=P_{\alpha_{g}}, & P_{\beta \alpha}=P_{\beta_{g}}, \quad P_{\alpha \beta}=0 & P_{\beta \beta}=0, \\
\bar{P}_{\alpha \alpha}=0 & \bar{P}_{\beta \alpha}=0, \quad \bar{P}_{\alpha \beta}=\bar{P}_{\beta_{g}^{-1}}, & \bar{P}_{\beta \beta}=\bar{P}_{\alpha_{g}^{-1}} .
\end{array}
$$

A direct calculation reveals that, up to multiplication by $L^{\times}$,

$$
\begin{array}{ll}
\widetilde{\operatorname{Reg}}_{\alpha \alpha}\left(E, V_{12}\right)=\log _{p}\left(\bar{P}_{\alpha_{g}^{-1}}\right) \cdot P, & \widetilde{\operatorname{Reg}}_{\alpha \beta}\left(E, V_{12}\right)=\log _{p}\left(P_{\beta_{g}}\right) \cdot \bar{P}, \\
\widetilde{\operatorname{Reg}}_{\beta \alpha}\left(E, V_{12}\right)=\log _{p}\left(\bar{P}_{\beta_{g}^{-1}}\right) \cdot P, & \widetilde{\operatorname{Reg}}_{\beta \beta}\left(E ; V_{12}\right)=\log _{p}\left(P_{\alpha_{g}}\right) \cdot \bar{P} .
\end{array}
$$

It follows that

$$
\begin{array}{ll}
\operatorname{Reg}_{g_{\alpha}}\left(E, V_{12}\right)=\log _{p}\left(\bar{P}_{\alpha_{g}^{-1}}\right) \cdot \log _{p}\left(P_{\beta_{g}}\right), & \operatorname{Reg}_{g_{\beta}}\left(E, V_{12}\right)=\log _{p}\left(\bar{P}_{\beta_{g}^{-1}}\right) \cdot \log _{p}\left(P_{\alpha_{g}}\right), \\
\operatorname{Reg}_{h_{\alpha}}\left(E, V_{12}\right)=0, & \operatorname{Reg}_{h_{\beta}}\left(E, V_{12}\right)=0 .
\end{array}
$$

as described in $[15, \$ 6]$. 


\subsection{Complex multiplication classes and Heegner points}

In this chapter we consider the setting where $g$ and $h$ are theta series attached to characters $\psi_{g}$ and $\psi_{h}$ of the same imaginary quadratic field $K$, and with inverse nebentypus character. Given any character $\psi$ of $G_{K}$, let $\psi^{\prime}$ denote the character obtained by conjugating it with the involution in $\mathrm{Gal}(K / \mathbb{Q})$. Then

$$
V_{g}=\operatorname{Ind}_{K}^{\mathbb{Q}} \psi_{g}=\operatorname{Ind}_{K}^{\mathbb{Q}} \psi_{g}^{\prime}, \quad V_{h}=\operatorname{Ind}_{K}^{\mathbb{Q}} \psi_{h}=\operatorname{Ind}_{K}^{\mathbb{Q}} \psi_{h}^{\prime},
$$

and therefore

$$
V_{g h}=\operatorname{Ind}_{K}^{Q} \psi_{\bullet} \oplus \operatorname{Ind}_{K}^{\mathbb{Q}} \psi_{0}, \quad \text { where } \psi_{\bullet}=\psi_{g} \psi_{h}, \quad \psi_{\circ}=\psi_{g} \psi_{h}^{\prime} .
$$

The self-duality assumption implies that $\psi_{\bullet}$ and $\psi_{\circ}$ are are ring class characters, i.e. they satisfy

$$
\psi_{\bullet}^{\prime}=\psi_{\bullet}^{-1}, \quad \psi_{\circ}^{\prime}=\psi_{\circ}^{-1} .
$$

Assume that the induced representations

$$
V_{\bullet}:=\operatorname{Ind}_{K}^{\mathbb{Q}} \psi_{\bullet}, \quad V_{\circ}:=\operatorname{Ind}_{K}^{\mathbb{Q}} \psi_{\circ}
$$

appearing in the decomposition

$$
V_{12}=V_{\bullet} \oplus V_{\circ}
$$

(viewed as representations with coefficients in the number field $L$ ) are irreducible, which is always the case unless $\psi_{\bullet}$ or $\psi_{0}$ is a quadratic, i.e. a genus character. (The more degenerate case where this arises can be subsumed under the "adjoint setting" considered in Sect. 4.5.)

The Hasse-Weil-Artin $L$-series

$$
L\left(E, V_{g h}, s\right)=L\left(E, V_{\bullet}, s\right) L\left(E, V_{0}, s\right)=L\left(E / K, \psi_{\bullet}, s\right) L\left(E / K, \psi_{0}, s\right)
$$

has a double zero at $s=1$ in one of the following two cases:

(1) The primitive $L$-series $L\left(E, V_{\bullet}, s\right)$ and $L\left(E, V_{0}, s\right)$ each have a simple zero at $s=1$. This setting, which resembles more closely the phenomena described in the previous two sections on Beilinson-Kato and Beilinson-Flach elements, will be referred to as the rank $(1,1)$ setting of Conjecture 3.12.

(2) Exactly one of the primitive $L$-series $L\left(E, V_{\bullet}, s\right)$ or $L\left(E, V_{\circ}, s\right)$ has a double zero at $s=1$, and the other is non-vanishing at the centre. This case shall be referred to as the rank $(2,0)$ setting of Conjecture 3.12. The possible non-triviality of the generalised Kato classes in the presence of a "genuine" double zero of a primitive Hasse-Weil-Artin Lfunction represents a novel feature that did not arise in the setting of Beilinson-Kato or Beilinson-Flach elements.

\subsubsection{The rank $(1,1)$ setting}

In this case, Conjectures $\operatorname{BSD}\left(E, V_{0}\right)$ and $\operatorname{BSD}\left(E, V_{0}\right)$ predict that the Mordell-Weil groups $\left(E(H)_{L} \otimes V_{\bullet}\right)^{G_{\mathbb{Q}}}$ and $\left(E(H)_{L} \otimes V_{\circ}\right)^{G_{\mathbb{Q}}}$ are both one-dimensional $L$-vector spaces, with generators $P_{\bullet}$ and $P_{0}$, respectively. It is natural to write

$$
P_{\bullet}=P_{\psi_{\bullet}} \otimes v_{\psi_{\bullet}^{\prime}}+P_{\psi_{\bullet}^{\prime}} \otimes v_{\psi_{\bullet}}, \quad P_{\circ}=P_{\psi_{0}} \otimes v_{\psi_{0}^{\prime}}+P_{\psi_{0}^{\prime}} \otimes v_{\psi_{0}},
$$

where $P_{\psi_{\bullet}}, P_{\psi_{\bullet}^{\prime}}, P_{\psi_{0}}$, and $P_{\psi_{0}^{\prime}}$ are generators for the one-dimensional subspaces of $E(H)_{L}$ on which $G_{K}$ acts via the characters $\psi_{\bullet}, \psi_{\bullet}^{\prime}, \psi_{\circ}$, and $\psi_{\circ}^{\prime}$, respectively. 
The description of the enhanced regulators attached to $V_{12}$ and to $\left(P_{\bullet}, P_{\circ}\right)$ can be further subdivided into two cases, with markedly different features: the case where the prime $p$ is split in $K$, and the case where it is inert in $K$.

a) The case where $p$ is split in $K$. In this case, let $p=\mathfrak{p} \mathfrak{p}^{\prime}$ be the factorisation of $p$ into distinct primes of $K$. We can then set

$$
\alpha_{g}=\psi_{g}(\mathfrak{p}), \quad \beta_{g}=\psi_{g}\left(\mathfrak{p}^{\prime}\right), \quad \alpha_{h}=\psi_{h}(\mathfrak{p}), \quad \beta_{h}=\psi_{h}\left(\mathfrak{p}^{\prime}\right),
$$

so that

$$
\alpha_{g} \alpha_{h}=\psi_{\bullet}(\mathfrak{p}), \quad \alpha_{g} \beta_{h}=\psi_{0}(\mathfrak{p}), \quad \beta_{g} \alpha_{h}=\psi_{\circ}\left(\mathfrak{p}^{\prime}\right), \quad \beta_{g} \beta_{h}=\psi_{\bullet}\left(\mathfrak{p}^{\prime}\right) .
$$

The decomposition of the $G_{K_{\mathfrak{p}}}=G_{\mathbb{Q}_{p}}$ representations attached to (35) into $\mathrm{Fr}_{p^{-}}$ eigenspaces is also stable under the action of the global Galois group $G_{K}$, and is described by:

$$
V_{12}^{\alpha \alpha}=V_{\bullet}^{\psi_{\bullet}}, \quad V_{12}^{\alpha \beta}=V_{\circ}^{\psi_{0}}, \quad V_{12}^{\beta \alpha}=V_{\circ}^{\psi_{0}^{\prime}}, \quad V_{12}^{\beta \beta}=V_{\bullet}^{\psi_{\bullet}^{\prime}} .
$$

It follows that, up to multiplication by $L^{\times}$,

$$
\begin{array}{ll}
\widetilde{\operatorname{Reg}}_{\alpha \alpha}\left(E, V_{12}\right)=\log _{\mathfrak{p}}\left(P_{\psi_{\bullet}^{\prime}}\right) \cdot P_{\circ}, & \widetilde{\operatorname{Reg}}_{\alpha \beta}\left(E, V_{12}\right)=\log _{\mathfrak{p}}\left(P_{\psi^{\prime}}\right) \cdot P_{\bullet}, \\
\widetilde{\operatorname{Reg}}_{\beta \alpha}\left(E, V_{12}\right)=\log _{\mathfrak{p}}\left(P_{\psi_{\bullet}}\right) \cdot P_{\bullet}, & \widetilde{\operatorname{Reg}}_{\beta \beta}\left(E, V_{12}\right)=\log _{\mathfrak{p}}\left(P_{\psi_{\bullet}}\right) \cdot P_{\circ},
\end{array}
$$

and therefore that

$$
\begin{array}{ll}
\operatorname{Reg}_{g_{\alpha}}\left(E, V_{12}\right)=\log _{\mathfrak{p}}\left(P_{\psi_{\iota^{\prime}}}\right) \cdot \log _{\mathfrak{p}}\left(P_{\psi_{\psi^{\prime}}}\right), & \operatorname{Reg}_{g_{\beta}}\left(E, V_{12}\right)=\log _{\mathfrak{p}}\left(P_{\psi_{\bullet}}\right) \cdot \log _{\mathfrak{p}}\left(P_{\psi_{0}}\right), \\
\operatorname{Reg}_{h_{\alpha}}\left(E, V_{12}\right)=\log _{\mathfrak{p}}\left(P_{\psi_{\bullet}^{\prime}}\right) \cdot \log _{\mathfrak{p}}\left(P_{\psi_{0}}\right), & \operatorname{Reg}_{h_{\beta}}\left(E, V_{12}\right)=\log _{\mathfrak{p}}\left(P_{\psi_{\bullet}}\right) \cdot \log _{\mathfrak{p}}\left(P_{\psi_{0}^{\prime}}\right) .
\end{array}
$$

The corresponding formulae for the $p$-adic iterated integrals $\mathscr{L}_{p}{ }^{g_{\alpha}}(f, g, h), \mathscr{L}_{p}{ }^{g_{\beta}}(f, g, h)$, $\mathscr{L}_{p}{ }^{h_{\alpha}}(f, g, h)$, and $\mathscr{L}_{p}{ }^{h_{\beta}}(f, g, h)$ were proved in [15, $\left.\$ 3\right]$, by using the $p$-adic Gross-Zagier formula of [8] to express these $L$-values in terms of products of $p$-adic logarithms of Heegner points. Theorem 3.3 of loc.cit. is one of the few pieces of theoretical evidence in support of Conjecture 3.12 .

b) The case where $p$ is inert in $K$. In this case, the eigenvalues of the Frobenius automorphism $\operatorname{Fr}_{p}$ acting on $V_{g}$ and $V_{h}$ are of the form

$$
\alpha_{g}, \quad \beta_{g}=-\alpha_{g}, \quad \alpha_{h}=\alpha_{g}^{-1}, \quad \beta_{h}=-\alpha_{g}^{-1} .
$$

Let $\left(v_{\psi_{g}}, v_{\psi_{g}^{\prime}}\right)$ be a eigenbasis of $V_{g}$ for the action of $G_{K}$ relative to the distinct characters $\psi_{g}$ and $\psi_{g}^{\prime}$, and let $\left(v_{\psi_{h}}, v_{\psi_{h}^{\prime}}\right)$ be a similar basis for $V_{h}$. These vectors can be scaled so that $\mathrm{Fr}_{p}$ acts on them as

$$
\begin{aligned}
& \operatorname{Fr}_{p}\left(v_{\psi_{g}}\right)=\alpha_{g} \cdot v_{\psi_{g}^{\prime}}, \quad \operatorname{Fr}_{p}\left(v_{\psi_{g}^{\prime}}\right)=\alpha_{g} \cdot v_{\psi_{g}}, \quad \operatorname{Fr}_{p}\left(v_{\psi_{h}}\right)=\alpha_{g}^{-1} \cdot v_{\psi_{h}^{\prime}}, \\
& \operatorname{Fr}_{p}\left(v_{\psi_{h}^{\prime}}\right)=\alpha_{g}^{-1} \cdot v_{\psi_{h}},
\end{aligned}
$$

and therefore we may set

$$
\begin{gathered}
V_{g}^{\alpha}=L \cdot\left(v_{\psi_{g}}+v_{\psi_{g}^{\prime}}\right), \quad V_{g}^{\beta}=L \cdot\left(v_{\psi_{g}}-v_{\psi_{g}^{\prime}}\right), \quad V_{h}^{\alpha}=L \cdot\left(v_{\psi_{h}}+v_{\psi_{h}^{\prime}}\right), \\
V_{h}^{\beta}=L \cdot\left(v_{\psi_{h}}-v_{\psi_{h}^{\prime}}\right) .
\end{gathered}
$$

After setting

$$
v_{\psi_{\bullet}}:=v_{\psi_{g}} \otimes v_{\psi_{h}}, \quad v_{\psi_{\bullet}^{\prime}}:=v_{\psi_{g}^{\prime}} \otimes v_{\psi_{h}^{\prime}}, \quad v_{\psi_{0}}:=v_{\psi_{g}} \otimes v_{\psi_{h}^{\prime}}, \quad v_{\psi_{0}^{\prime}}:=v_{\psi_{g}^{\prime}} \otimes v_{\psi_{h}},
$$

and letting

$$
v_{\bullet}^{+}:=v_{\psi_{\bullet}}+v_{\psi_{\bullet}^{\prime}} \quad v_{\bullet}^{-}:=v_{\psi_{\bullet}}-v_{\psi_{\bullet}^{\prime}}, \quad v_{\circ}^{+}:=v_{\psi_{0}}+v_{\psi_{0}^{\prime}} \quad v_{\bullet}^{-}:=v_{\psi_{0}}-v_{\psi_{0}^{\prime}},
$$


it is easy to see that

$$
\begin{array}{lll}
V_{12}^{\alpha \alpha}=L \cdot\left(v_{\bullet}^{+}+v_{\circ}^{+}\right), & & V_{12}^{\alpha \beta}=L \cdot\left(v_{\bullet}^{-}+v_{\circ}^{-}\right), \\
V_{12}^{\beta \alpha}=L \cdot\left(v_{\bullet}^{-}-v_{\circ}^{-}\right), & & V_{12}^{\beta \beta}=L \cdot\left(v_{\bullet}^{+}-v_{\circ}^{+}\right) .
\end{array}
$$

Note that $\operatorname{Fr}_{p}$ acts on both $V_{12}^{\alpha \alpha}$ and $V_{12}^{\beta \beta}$ with the eigenvalue 1 , and on $V_{12}^{\alpha \beta}$ and $V_{12}^{\beta \alpha}$ with the eigenvalue -1 . In particular, while $V_{g}$ and $V_{h}$ are always regular at $p$, the tensor product $V_{g h} \simeq V_{12}$ never enjoys this property, even though the vector spaces described in (37) are one-dimensional.

Keeping the same notations as in (36), let

$$
P_{\bullet}^{+}:=P_{\psi_{\bullet}}+P_{\psi_{\bullet}^{\prime}}, \quad P_{\bullet}^{-}:=P_{\psi_{\bullet}}-P_{\psi_{\bullet}^{\prime}}, \quad P_{\circ}^{+}:=P_{\psi_{0}}+P_{\psi_{0}^{\prime}}, \quad P_{\circ}^{-}:=P_{\psi_{0}}-P_{\psi_{0}^{\prime}} .
$$

With these notations, the enhanced regulators describing the associated generalised Kato classes are given by

$$
\begin{array}{ll}
\widetilde{\operatorname{Reg}}_{\alpha \alpha}\left(E, V_{12}\right)=\log _{p}\left(P_{\bullet}^{+}\right) \cdot P_{\circ}-\log _{p}\left(P_{\circ}^{+}\right) \cdot P_{\bullet}, & \widetilde{\operatorname{Reg}}_{\alpha \beta}\left(E, V_{12}\right)=\log _{p}\left(P_{\bullet}^{-}\right) \cdot P_{\circ}-\log _{p}\left(P_{\circ}^{-}\right) \cdot P_{\bullet} \\
\widetilde{\operatorname{Reg}}_{\beta \alpha}\left(E, V_{12}\right)=\log _{p}\left(P_{\bullet}^{-}\right) \cdot P_{\circ}+\log _{p}\left(P_{\circ}^{-}\right) \cdot P_{\bullet}, & \widetilde{\operatorname{Reg}}_{\beta \beta}\left(E, V_{12}\right)=\log _{p}\left(P_{\bullet}^{+}\right) \cdot P_{\circ}+\log _{p}\left(P_{\circ}^{+}\right) \cdot P_{\bullet}
\end{array}
$$

The four regulators $\operatorname{Reg}_{g_{\alpha}}\left(E, V_{12}\right), \operatorname{Reg}_{g_{\beta}}\left(E, V_{12}\right), \operatorname{Reg}_{h_{\alpha}}\left(E, V_{12}\right)$, and $\operatorname{Reg}_{h_{\beta}}\left(E, V_{12}\right)$ are all seen to be simple $L^{\times}$-multiples of the expression

$$
\log _{p}\left(P_{\bullet}^{+}\right) \cdot \log _{p}\left(P_{\circ}^{-}\right)-\log _{p}\left(P_{\circ}^{+}\right) \cdot \log _{p}\left(P_{\bullet}^{-}\right) .
$$

The resulting formula for $\mathscr{L}_{p}{ }^{g_{\alpha}}(f, g, h)$ predicted by Conjecture 3.8 has been extensively tested numerically in $[15, \$ 3.3]$.

Remark 4.1 Even though the points $P_{\bullet}^{+}, P_{\bullet}^{-}, P_{\circ}^{+}$, and $P_{\circ}^{-}$that figure in the generalised Kato classes are in principle expressed as linear combinations of Heegner points, the methods used to prove Conjecture 3.8 when $p$ is split in $K$, which are based on the $p$-adic Gross-Zagier formula of [8] and on properties of the Katz $p$-adic $L$-function attached to $K$, seem to break down completely when $p$ is inert in $K$. A theoretical understanding of the $p$-adic iterated integrals of [15] in this setting would seem to require a new idea.

Remark 4.2 It is worth contrasting the expressions arising in (38) with the simpler formulae

$$
\begin{aligned}
& \log _{\alpha \alpha} \widetilde{\operatorname{Reg}}_{\beta \beta}\left(E, V_{12}\right)=\log _{\beta \beta} \widetilde{\operatorname{Reg}}_{\alpha \alpha}\left(E, V_{12}\right)=\log _{p}\left(P_{\bullet}^{+}\right) \cdot \log _{p}\left(P_{\circ}^{+}\right), \\
& \log _{\alpha \beta} \widetilde{\operatorname{Reg}}_{\beta \alpha}\left(E, V_{12}\right)=\log _{\beta \alpha} \widetilde{\operatorname{Reg}}_{\alpha \beta}\left(E, V_{12}\right)=\log _{p}\left(P_{\bullet}^{-}\right) \cdot \log _{p}\left(P_{\circ}^{-}\right) .
\end{aligned}
$$

In certain very special settings-notably, when the elliptic curve $E$ has multiplicative reduction at $p$-these expressions arise as the leading terms of the $p$-adic $L$-series

$$
\mathscr{L}_{p}^{f}\left(f, g_{\alpha}, h_{\alpha}\right), \quad \ldots, \quad \mathscr{L}_{p}^{f}\left(f, g_{\beta}, h_{\beta}\right) .
$$

Methods based on the Cerednik-Drinfeld theory of $p$-adic uniformisation of Shimura curves make it possible to relate these leading terms to the $p$-adic logarithms of Heegner points, leading to some indirect theoretical evidence for Conjecture 3.12 in the setting where $p$ is inert in $K$. See [20] for a description of this approach.

\subsubsection{The rank $(2,0)$ setting}

Assume, after possibly interchanging $V_{\bullet}$ and $V_{0}$, that $L\left(E, V_{\bullet}, s\right)$ has a double zero at $s=1$ and that $L\left(E, V_{\circ}, 1\right) \neq 0$. In this case, Conjectures $\operatorname{BSD}\left(E, V_{\bullet}\right)$ and $\operatorname{BSD}\left(E, V_{\circ}\right)$ predict that 


$$
\left(E(H)_{L} \otimes V_{12}\right)^{G_{\mathbb{Q}}}=\left(E(H)_{L} \otimes V_{\bullet}\right)^{G_{\mathbb{Q}}}
$$

is two-dimensional over $L$. Choose a basis $(P, Q)$ for this vector space. It is natural to write

$$
P=P_{\psi_{\bullet}} \otimes v_{\psi_{\bullet}^{\prime}}+P_{\psi_{\bullet}^{\prime}} \otimes v_{\psi_{\bullet}}, \quad Q=Q_{\psi_{\bullet}} \otimes v_{\psi_{\bullet}^{\prime}}+Q_{\psi_{\bullet}^{\prime}} \otimes v_{\psi_{\bullet}}
$$

where $\left(P_{\psi_{\bullet}}, Q_{\psi_{\bullet}}\right)$ and $\left(P_{\psi_{\bullet}^{\prime}}, Q_{\psi_{\bullet}^{\prime}}\right)$ are bases for the two-dimensional subspaces of $E(H)_{L}$ on which $G_{K}$ acts via the characters $\psi_{\bullet}$ and $\psi_{\bullet}^{\prime}$, respectively.

As in the rank $(1,1)$ setting, the shape of the enhanced regulators attached to $V_{12}$ and to the basis $(P, Q)$ depend very much on whether the prime $p$ is split or inert in $K$.

a) The case where $p$ is split in $K$. In this case, letting $p=\mathfrak{p} \mathfrak{p}^{\prime}$ be the factorisation of $p$ into distinct primes of $K$, we can set

$$
\alpha_{g}=\psi_{g}(\mathfrak{p}), \quad \beta_{g}=\psi_{g}\left(\mathfrak{p}^{\prime}\right), \quad \alpha_{h}=\psi_{h}(\mathfrak{p}), \quad \beta_{h}=\psi_{h}\left(\mathfrak{p}^{\prime}\right),
$$

so that

$$
\alpha_{g} \alpha_{h}=\psi_{\bullet}(\mathfrak{p}), \quad \alpha_{g} \beta_{h}=\psi_{\circ}(\mathfrak{p}), \quad \beta_{g} \alpha_{h}=\psi_{\circ}\left(\mathfrak{p}^{\prime}\right), \quad \beta_{g} \beta_{h}=\psi_{\bullet}\left(\mathfrak{p}^{\prime}\right) .
$$

The decomposition of the $G_{K_{\mathfrak{p}}}=G_{\mathbb{Q}_{p}}$ representations attached to (35) into $\mathrm{Fr}_{p^{-}}$ eigenspaces is also stable under the action of the global Galois group $G_{K}$ and is described by:

$$
V_{12}^{\alpha \alpha}=V_{\bullet}^{\psi_{\bullet}}, \quad V_{12}^{\alpha \beta}=V_{\circ}^{\psi_{0}}, \quad V_{12}^{\beta \alpha}=V_{\circ}^{\psi_{0}^{\prime}}, \quad V_{12}^{\beta \beta}=V_{\bullet}^{\psi_{\bullet}^{\prime}} .
$$

It follows that, up to multiplication by $L^{\times}$,

$$
\begin{array}{ll}
\widetilde{\operatorname{Reg}}_{\alpha \alpha}\left(E, V_{12}\right)=\log _{\mathfrak{p}}\left(P_{\psi^{\prime}}\right) \cdot Q-\log _{\mathfrak{p}}\left(Q_{\psi_{\bullet}^{\prime}}\right) \cdot P, & \widetilde{\operatorname{Reg}}_{\alpha \beta}\left(E, V_{12}\right)=0, \\
\widetilde{\operatorname{Reg}}_{\beta \alpha}\left(E, V_{12}\right)=0, & \widetilde{\operatorname{Reg}}_{\beta \beta}\left(E, V_{12}\right)=\log _{\mathfrak{p}}\left(P_{\psi_{\bullet}}\right) \cdot Q-\log _{\mathfrak{p}}\left(Q_{\psi_{\mathfrak{\bullet}}}\right) \cdot P .
\end{array}
$$

This suggests that the generalised Kato classes $\kappa\left(f, g_{\alpha}, h_{\alpha}\right)$ and $\kappa\left(f, g_{\beta}, h_{\beta}\right)$ give non-trivial elements of the two-dimensional vector space $\left(E(H)_{L} \otimes V_{\bullet}\right)^{G}$, while the generalised Kato classes $\kappa\left(f, g_{\alpha}, h_{\beta}\right)$ and $\kappa\left(f, g_{\beta}, h_{\alpha}\right)$ should vanish. Furthermore, a direct calculation shows that

$$
\operatorname{Reg}_{g_{\alpha}}\left(E, V_{12}\right)=\operatorname{Reg}_{g_{\beta}}\left(E, V_{12}\right)=\operatorname{Reg}_{h_{\alpha}}\left(E, V_{12}\right)=\operatorname{Reg}_{h_{\beta}}\left(E, V_{12}\right)=0,
$$

which is consistent with the fact, proved in $[15, \$ 3.2]$, that all the $p$-adic iterated integrals attached to $(f, g, h)$ vanish in the rank $(2,0)$ setting when $p$ is split in $K$. In this case the generalised Kato classes carry more arithmetic information that the $p$-adic iterated integrals which describe (certain of) their $p$-adic logarithms. This represents yet another setting where Conjecture 3.12 is a genuine strengthening of Conjecture 3.8 of [15].

b) The case where $p$ is inert in $K$. After scaling the points $P_{\psi_{\bullet}}$, and $P_{\psi_{\bullet}^{\prime}}, Q_{\psi_{\bullet}}$, and $Q_{\psi_{\bullet}^{\prime}}$ in such a way that

$$
\operatorname{Fr}_{p}\left(P_{\psi_{\bullet}}\right)=P_{\psi_{\bullet}^{\prime}}, \quad \operatorname{Fr}_{p}\left(P_{\psi_{\bullet}^{\prime}}\right)=P_{\psi_{\bullet}}, \quad \operatorname{Fr}_{p}\left(Q_{\psi_{\bullet}}\right)=Q_{\psi_{\bullet}^{\prime}}, \quad \operatorname{Fr}_{p}\left(Q_{\psi_{\bullet}^{\prime}}\right)=Q_{\psi_{\bullet}}
$$

and letting

$$
P^{ \pm}:=P_{\psi_{\bullet}} \pm P_{\psi_{\bullet}^{\prime}} \quad Q^{ \pm}:=Q_{\psi_{\bullet}} \pm Q_{\psi_{\bullet}^{\prime}},
$$

the enhanced regulators are given by

$$
\begin{array}{ll}
\widetilde{\operatorname{Reg}}_{\alpha \alpha}\left(E, V_{12}\right)=\log _{p}\left(P^{+}\right) \cdot Q-\log _{p}\left(Q^{+}\right) \cdot P, & \widetilde{\operatorname{Reg}}_{\alpha \beta}\left(E, V_{12}\right)=\log _{p}\left(P^{-}\right) \cdot Q-\log _{p}\left(Q^{-}\right) \cdot P, \\
\widetilde{\operatorname{Reg}}_{\beta \alpha}\left(E, V_{12}\right)=\log _{p}\left(P^{-}\right) \cdot Q-\log _{p}\left(Q^{-}\right) \cdot P, & \widetilde{\operatorname{Reg}}_{\beta \beta}\left(E, V_{12}\right)=\log _{p}\left(P^{+}\right) \cdot Q-\log _{p}\left(Q^{+}\right) \cdot P .
\end{array}
$$


In this case, the four regulators $\operatorname{Reg}_{g_{\alpha}}\left(E, V_{12}\right), \ldots, \operatorname{Reg}_{h_{\beta}}\left(E, V_{12}\right)$ attached to $(f, g, h)$ are explicit multiples of the expression

$$
\log _{p}\left(P^{+}\right) \cdot \log _{p}\left(Q^{-}\right)-\log _{p}\left(Q^{+}\right) \cdot \log _{p}\left(P^{-}\right) .
$$

See [15, Ex. 3.14] for some numerical verifications of the agreement between this value and the $p$-adic iterated integrals attached to $(f, g, h)$.

Equations (39) and (40) combined with Conjecture 3.12 suggest that the generalised Kato classes always generate the Mordell-Weil group $\left(E(H)_{L} \otimes V_{\bullet}\right)^{G_{\mathbb{Q}}}$ (tensored over $L$ with $\left.\mathbb{Q}_{p}\right)$ in the rank $(2,0)$ setting. Since the irreducible representation $V_{\bullet}$ occurs with multiplicity two in $E(H)_{L}$, none of the $V_{\bullet}$-isotypic part of the Mordell-Weil group is expected to be accounted for by Heegner points, as discussed in the introduction.

\subsection{Real multiplication classes and Stark-Heegner points}

In this chapter we consider the setting where $g$ and $h$ are theta series attached to characters $\psi_{g}$ and $\psi_{h}$ of mixed signature of the same real quadratic field $K$. In that case, we have, exactly as in Sect. 4.3,

$$
V_{g}=\operatorname{Ind}_{K}^{\mathbb{Q}} \psi_{g}, \quad V_{h}=\operatorname{Ind}_{K}^{\mathbb{Q}} \psi_{h},
$$

and

$$
V_{g h}=V_{\bullet} \oplus V_{\circ}:=\operatorname{Ind}_{K}^{\mathbb{Q}} \psi_{\bullet} \oplus \operatorname{Ind}_{K}^{\mathbb{Q}} \psi_{\circ}, \quad \text { where } \psi_{\bullet}=\psi_{g} \psi_{h}, \quad \psi_{\circ}=\psi_{g} \psi_{h}^{\prime} .
$$

The characters $\psi_{0}$ and $\psi_{0}$ are also ring class characters of $K$, with one totally even, and the other totally odd. Once again, it is convenient to assume that $V_{\bullet}$ and $V_{\circ}$ are both irreducible, i.e. that neither $\psi_{\bullet}$ nor $\psi_{\circ}$ is a genus character of $K$.

As in the case where $K$ is imaginary, the study of the generalised Kato classes divides naturally into the rank $(1,1)$ and rank $(2,0)$ settings, depending on the orders of vanishing of $L\left(E / K, \psi_{\bullet}, s\right)$ and $L\left(E / K, \psi_{\circ}, s\right)$ (or, alternately, on the dimensions of $\left(E(H)_{L} \otimes V_{\bullet}\right)^{G_{\mathbb{Q}}}$ and $\left.\left(E(H)_{L} \otimes V_{\circ}\right)^{G_{\mathbb{Q}}}\right)$, and continue to depend in a crucial way on whether $p$ is split or inert in $K$. In all four cases, the formulae for the enhanced regulators are identical to those obtained in Sect. 4.3, so it is unecessary to reproduce them here, contenting ourselves with the following comments in connection with the rank $(1,1)$ setting.

a) The case where $p$ is split in $K$. This setting, where the greatest amount of theoretical evidence was available when $K$ is imaginary quadratic, thanks to the theory of Heegner points, is a lot more mysterious when $K$ is real quadratic. With notations being the same as in Sect. 4.3.1, we have

$$
\begin{array}{ll}
\widetilde{\operatorname{Reg}}_{\alpha \alpha}\left(E, V_{12}\right)=\log _{\mathfrak{p}}\left(P_{\psi_{\bullet}}\right) \cdot P_{\circ}, & \widetilde{\operatorname{Reg}}_{\alpha \beta}\left(E, V_{12}\right)=\log _{\mathfrak{p}}\left(P_{\psi_{0}^{\prime}}\right) \cdot P_{\bullet}, \\
\widetilde{\operatorname{Reg}}_{\beta \alpha}\left(E, V_{12}\right)=\log _{\mathfrak{p}}\left(P_{\psi_{\mathfrak{o}}}\right) \cdot P_{\bullet}, & \widetilde{\operatorname{Reg}}_{\beta \beta}\left(E, V_{12}\right)=\log _{\mathfrak{p}}\left(P_{\psi_{\bullet}}\right) \cdot P_{\mathrm{o}},
\end{array}
$$

and

$$
\begin{array}{ll}
\operatorname{Reg}_{g_{\alpha}}\left(E, V_{12}\right)=\log _{\mathfrak{p}}\left(P_{\psi_{\bullet}^{\prime}}\right) \cdot \log _{\mathfrak{p}}\left(P_{\psi_{0}^{\prime}}\right), \quad \operatorname{Reg}_{g_{\beta}}\left(E, V_{12}\right)=\log _{\mathfrak{p}}\left(P_{\psi_{\mathfrak{\bullet}}}\right) \cdot \log _{\mathfrak{p}}\left(P_{\psi_{0}}\right), \\
\operatorname{Reg}_{h_{\alpha}}\left(E, V_{12}\right)=\log _{\mathfrak{p}}\left(P_{\psi_{\bullet}^{\prime}}\right) \cdot \log _{\mathfrak{p}}\left(P_{\psi_{0}}\right), & \operatorname{Reg}_{h_{\beta}}\left(E, V_{12}\right)=\log _{\mathfrak{p}}\left(P_{\psi_{\mathfrak{\bullet}}}\right) \cdot \log _{\mathfrak{p}}\left(P_{\psi_{0}^{\prime}}\right) .
\end{array}
$$

This setting has special appeal in connection with an eventual (for now, highly conjectural, and not even clearly formulated) theory of Stark-Heegner points over ring class fields of real quadratic fields. It would be of great interest to relate (conjecturally, at least) the regulators in (42) and in (43) to generalised Kato classes and $p$-adic iterated integrals, respectively. 
The obstruction to doing this is that the modular forms $g$ and $h$ (more precisely, their stabilisations) fail to obey Hypothesis IV in Sect. 2. When $g$ is a modular form of RM type which is regular at a prime $p$ which splits in $K$, the Stark unit $u_{g_{\alpha}}$ is also unavailable, and an analogue of Conjecture 3.8 has yet to be formulated precisely in this setting. Because of the tantalising connection with Stark-Heegner points defined over ring class fields of $K$, it would be of great interest to extend the Conjectures of [15], as well as Conjecture 3.12, to the real quadratic context. A first step has been made in [17] towards understanding the periods of $\mathbb{} 2$ in this setting.

b) The case where $p$ is inert in $K$. The formulae for the enhanced regulators are identical to those in Part b) of Sect. 4.3.1, namely:

$$
\begin{array}{ll}
\widetilde{\operatorname{Reg}}_{\alpha \alpha}\left(E, V_{12}\right)=\log _{p}\left(P_{\bullet}^{+}\right) \cdot P_{\circ}-\log _{p}\left(P_{\circ}^{+}\right) \cdot P_{\bullet}, & \widetilde{\operatorname{Reg}}_{\alpha \beta}\left(E, V_{12}\right)=\log _{p}\left(P_{\bullet}^{-}\right) \cdot P_{\circ}-\log _{p}\left(P_{\circ}^{-}\right) \cdot P_{\bullet}, \\
\widetilde{\operatorname{Reg}}_{\beta \alpha}\left(E, V_{12}\right)=\log _{p}\left(P_{\bullet}^{-}\right) \cdot P_{\circ}+\log _{p}\left(P_{\circ}^{-}\right) \cdot P_{\bullet}, & \widetilde{\operatorname{Reg}}_{\beta \beta}\left(E, V_{12}\right)=\log _{p}\left(P_{\bullet}^{+}\right) \cdot P_{\circ}+\log _{p}\left(P_{\circ}^{+}\right) \cdot P_{\bullet} .
\end{array}
$$

The $p$-adic logarithms of these enhanced regulators ought to involve linear combinations of products of logarithms of so-called Stark-Heegner points. This prediction has been extensively tested numerically in $[15, \$ 4.2]$.

Remark 4.3 The logarithms of the generalised Kato classes that are not amenable to expressions in terms of $p$-adic iterated integrals are expected to admit particularly simple expressions, as suggested by the formulae

$$
\begin{aligned}
& \log _{\alpha \alpha} \widetilde{\operatorname{Reg}}_{\beta \beta}\left(E, V_{12}\right)=\log _{\beta \beta} \widetilde{\operatorname{Reg}}_{\alpha \alpha}\left(E, V_{12}\right)=\log _{p}\left(P_{\bullet}^{+}\right) \cdot \log _{p}\left(P_{\circ}^{+}\right), \\
& \log _{\alpha \beta} \widetilde{\operatorname{Reg}}_{\beta \alpha}\left(E, V_{12}\right)=\log _{\beta \alpha} \widetilde{\operatorname{Reg}}_{\alpha \beta}\left(E, V_{12}\right)=\log _{p}\left(P_{\bullet}^{-}\right) \cdot \log _{p}\left(P_{\circ}^{-}\right) .
\end{aligned}
$$

In the special case where $E$ has multiplicative reduction at $p$, the article [20] in progress proves a formula of the shape

$$
\log _{\alpha \alpha} \kappa\left(f, g_{\alpha}, h_{\alpha}\right)=\log _{p}\left(P_{\bullet}^{? \pm}\right) \cdot \log _{p}\left(P_{\circ}^{? \pm}\right),
$$

where $P_{\bullet}^{? \pm}$ and $P_{\circ}^{? \pm}$ are the Stark-Heegner points of [14], and the sign that arises depends on whether $E$ has split or non-split multiplicative reduction at $p$.

\subsection{Adjoint classes}

The case where $h$ is dual to $g$ is of considerable arithmetic interest, since in that case the representation

$$
V_{g h}=\mathbb{Q}_{p} \oplus M_{g}, \quad\left(\text { where } M_{g}:=\operatorname{Ad}^{0}\left(V_{g}\right)\right),
$$

admits the trivial representation as a constituent. The generalised Kato classes attached to $g$ and $h$ may then, in appropriate circumstances, contribute to the Mordell-Weil group $E(\mathbb{Q})$, and it is interesting to understand when this occurs.

The Hasse-Weil-Artin $L$-series

$$
L\left(E, V_{g h}, s\right)=L(E, s) L\left(E, M_{g}, s\right)
$$

has a double zero at $s=1$ in one of the following three cases:

(1) the rank $(0,2)$ setting where $L(E, 1) \neq 0$ and $L\left(E, M_{g}, s\right)$ has a double zero at $s=1$;

(2) the $\operatorname{rank}(1,1)$ case where $L(E, s)$ and $L\left(E, M_{g}, s\right)$ each vanish to order 1 at $s=1$;

(3) the $\operatorname{rank}(2,0)$ setting where $L(E, s)$ has a double zero at the centre and $L\left(E, M_{g}, 1\right) \neq 0$. This case is particularly intriguing for its direct connection with the arithmetic of elliptic curves of rank two over $\mathbb{Q}$. 
In all the examples that will be treated below, we always set

$$
\alpha_{h}=\alpha_{g}^{-1}, \quad \beta_{h}=\beta_{g}^{-1},
$$

so that

$$
\alpha_{g} \alpha_{h}=1, \quad \alpha_{g} \beta_{h}=\alpha_{g} / \beta_{g}, \quad \beta_{g} \alpha_{h}=\frac{\beta_{g}}{\alpha_{g}}, \quad \beta_{g} \beta_{h}=1 .
$$

\subsubsection{The rank $(0,2)$ setting}

Let $(P, Q)$ be an $L$-basis for $\left(E(H)_{L} \otimes V_{12}\right)^{G_{\mathbb{Q}}}=\left(E(H)_{L} \otimes M_{g}\right)^{G_{\mathbb{Q}}}$, and write

$$
\begin{aligned}
& P=P_{\frac{\alpha}{\beta}} \otimes v_{\beta / \alpha}+P_{1} \otimes v_{1}+P_{\frac{\beta}{\alpha}} \otimes v_{\alpha / \beta}, \\
& Q=Q_{\frac{\alpha}{\beta}} \otimes v_{\beta / \alpha}+Q_{1} \otimes v_{1}+Q_{\frac{\beta}{\alpha}} \otimes v_{\alpha / \beta},
\end{aligned}
$$

where $v_{\beta / \alpha}, v_{1}$, and $v_{\alpha / \beta}$ are bases for the $\mathrm{Fr}_{p}$-eigenspaces of $W_{g}$ attached to the eigenvalues $\frac{\beta_{g}}{\alpha_{g}}, 1$, and $\alpha_{g} / \beta_{g}$, respectively. Then the four enhanced regulators are given, up to $L^{\times}$, as follows:

$$
\begin{array}{ll}
\widetilde{\operatorname{Reg}}_{\alpha \alpha}\left(E, V_{12}\right)=\log _{p}\left(P_{1}\right) \cdot Q-\log _{p}\left(Q_{1}\right) \cdot P, & \widetilde{\operatorname{Reg}}_{\alpha \beta}\left(E, V_{12}\right)=\log _{p}\left(P_{\frac{\beta}{\alpha}}\right) \cdot Q-\log _{p}\left(Q_{\frac{\beta}{\alpha}}\right) \cdot P, \\
\widetilde{\operatorname{Reg}}_{\beta \alpha}\left(E, V_{12}\right)=\log _{p}\left(P_{\frac{\alpha}{\beta}}\right) \cdot Q-\log _{p}\left(Q_{\frac{\alpha}{\beta}}\right) \cdot P, & \widetilde{\operatorname{Reg}}_{\beta \beta}\left(E, V_{12}\right)=\log _{p}\left(P_{1}\right) \cdot Q-\log _{p}\left(Q_{1}\right) \cdot P .
\end{array}
$$

Conjecture 3.12 suggests in this case that the generalised Kato class $\kappa\left(f, g_{\alpha}, h_{\beta}\right)$ generates the kernel of the map $\log _{\alpha \beta}$ in the two-dimensional $\mathbb{Q}_{p}$-vector space $\left(E(H)_{L} \otimes M_{g}\right)^{G_{\mathbb{Q}}} \otimes_{L}$ $\mathbb{Q}_{p}$.

We refer the reader to [15, Example 5.4] for the numerical verification of Conjecture 3.8 for two different instances in this setting.

\subsubsection{The rank $(1,1)$ setting}

Let $P$ be a generator of $E(\mathbb{Q})_{L}$ and let $Q$ be a generator of $\left(E(H)_{L} \otimes M_{g}\right)^{G_{\mathbb{Q}}}$. With the same notational conventions as before, we find:

$$
\begin{array}{ll}
\widetilde{\operatorname{Reg}}_{\alpha \alpha}\left(E, V_{12}\right)=\log _{p}(P) \cdot Q-\log _{p}\left(Q_{1}\right) \cdot P, & \widetilde{\operatorname{Reg}}_{\alpha \beta}\left(E, V_{12}\right)=\log _{p}\left(Q_{\frac{\beta}{\alpha}}\right) \cdot P, \\
\widetilde{\operatorname{Reg}}_{\beta \alpha}\left(E, V_{12}\right)=\log _{p}\left(Q_{\frac{\alpha}{\beta}}\right) \cdot P, & \widetilde{\operatorname{Reg}}_{\beta \beta}\left(E, V_{12}\right)=\log _{p}(P) \cdot Q-\log _{p}\left(Q_{1}\right) \cdot P .
\end{array}
$$

In contrast, we have

$$
\begin{array}{ll}
\operatorname{Reg}_{g_{\alpha}}\left(E, V_{12}\right)=\log _{p}(P) \cdot \log _{p}\left(Q_{\frac{\beta}{\alpha}}\right) & \operatorname{Reg}_{g_{\beta}}\left(E, V_{12}\right)=\log _{p}(P) \cdot \log _{p}\left(Q_{\frac{\alpha}{\beta}}\right), \\
\operatorname{Reg}_{h_{\alpha}}\left(E, V_{12}\right)=\log _{p}(P) \log _{p}\left(Q_{\frac{\alpha}{\beta}}\right), & \operatorname{Reg}_{h_{\beta}}\left(E, V_{12}\right)=\log _{p}(P) \cdot \log _{p}\left(Q_{\frac{\beta}{\alpha}}\right) .
\end{array}
$$

Many numerical examples where the $p$-adic iterated integrals attached to $(f, g, h)$ are seen to agree with these regulators are described in $[15, \$ 5]$. It is worth noting that the expression $\log _{p}\left(Q_{1}\right)$ that appears in the enhanced regulators of (44) disappears from the regulators (45) that arose in [15].

\subsubsection{The rank $(2,0)$ setting}

Let $(P, Q)$ be an $L$-basis of the two-dimensional $L$-vector space $E(\mathbb{Q})_{L}$. With the same notational conventions as before, we find:

$$
\begin{array}{ll}
\widetilde{\operatorname{Reg}}_{\alpha \alpha}\left(E, V_{12}\right)=\log _{p}(P) \cdot Q-\log _{p}(Q) \cdot P, & \widetilde{\operatorname{Reg}}_{\alpha \beta}\left(E, V_{12}\right)=0, \\
\widetilde{\operatorname{Reg}}_{\beta \alpha}\left(E, V_{12}\right)=0, & \widetilde{\operatorname{Reg}}_{\beta \beta}\left(E, V_{12}\right)=\log _{p}(P) \cdot Q-\log _{p}(Q) \cdot P .
\end{array}
$$


In other words, the generalised Kato classes $\kappa\left(f, g_{\alpha}, h_{\alpha}\right)$ and $\kappa\left(f, g_{\beta}, h_{\beta}\right)$ give (essentially, the same, up to $L^{\times}$-multiples) canonical element of $E(\mathbb{Q})_{\mathbb{Q}_{p}}$, which is expected to be non-trivial precisely when

$$
L^{\prime \prime}(E, 1) \neq 0, \quad L\left(E, M_{g}, 1\right) \neq 0 .
$$

Note that on the other hand

$$
\operatorname{Reg}_{g_{\alpha}}\left(E, V_{12}\right)=\operatorname{Reg}_{g_{\beta}}\left(E, V_{12}\right)=\operatorname{Reg}_{h_{\alpha}}\left(E, V_{12}\right)=\operatorname{Reg}_{h_{\beta}}\left(E, V_{12}\right)=0 .
$$

This last example gives yet another instance where Conjecture 3.12 represents a genuine strengthening of the elliptic Stark conjectures of [15]. It predicts that that generalised Kato classes of the form $\kappa\left(f, g_{\alpha}, \bar{g}_{1 / \alpha}\right)$ ought to give non-trivial elements in the pro- $p$-Selmer groups of elliptic curves of rank two over $\mathbb{Q}$, when the auxiliary $L$-value $L\left(E, M_{g}, 1\right)$ is nonzero. Testing this prediction experimentally seems to present an interesting challenge.

\section{Author details}

${ }^{1}$ Department of Mathematics and Statistics, McGill University, 805 Sherbrooke St. West, Montreal, Canada, ${ }^{2}$ Departament de Matemàtiques, Universitat Politècnica de Catalunya, C. Jordi Girona 1-3, 08034 Barcelona, Spain.

\section{Acknowledgements}

The first author was supported by an NSERC Discovery Grant, and the second author was supported by Grant MTM2012-34611.

Received: 3 July 2015 Accepted: 4 February 2016

Published online: 24 August 2016

\section{References}

1. Bellaïche, J., Dimitrov, M.: On the eigencurve at classical weight 1 points. Duke Math. J. 165(2), 245-266 (2016)

2. Bertolini, M., Castella, F., Darmon, H., Dasgupta, S., Prasanna, K., Rotger, V.: p-adic L-functions and Euler systems: a tale in two trilogies. In: Proceedings of the 2011 Durham Symposium on Automorphic Forms and Galois Representations, London Mathematical Society Lecture Notes, vol. 414, pp. 52-101 (2014)

3. Bertolini, M., Darmon, H.: A rigid analytic Gross-Zagier formulaand arithmetic applications (with an appendix by B. Edixhoven), Ann. Math. (2) 146(1), 111-147 (1997)

4. Bertolini, M., Darmon, H.: Euler systems and Jochnowitz congruences. Am. J. Math. 121, 259-281 (1999)

5. Bertolini, M., Darmon, H.: Iwasawa's main conjecture for elliptic curves over anticyclotomic $\mathbb{Z}_{p}$-extensions. Ann. Math. (2) 162(1), 1-64 (2005)

6. Bertolini, M., Darmon, H.: Kato's Euler system and rational points on elliptic curves I: A p-adic Beilinson formula. Isr. J. Math. 199(1), 163-188 (2014)

7. Bertolini, M., Darmon, H., Dasgupta, S.: Stark-Heegner points and special values of L-series. In: Burns, D., Buzzard, K., Nekovar, J. (eds.) L-functions and Galois Representations, London Mathematical Society LNS, vol. 320, pp. 1-23 (2007)

8. Bertolini, M., Darmon, H., Prasanna, K.: Generalised Heegner cycles and p-adic Rankin L-series (with an appendix by Brian Conrad). Duke Math. J. 162(6), 1033-1148 (2013)

9. Bertolini, M., Darmon, H., Prasanna, K.: Chow-Heegner points on CM elliptic curves and values of $p$-adic L-series. Int. Math. Res. Not. 2014, 745-793 (2014)

10. Bertolini, M., Darmon, H., Rotger, V.: Beilinson-Flach elements and Euler Systems I: syntomic regulators and $p$-adic Rankin L-series. J. Algebr. Geom. 24, 355-378 (2015)

11. Bertolini, M., Darmon, H., Rotger, V.: Beilinson-Flach elements and Euler systems II: the Birch and Swinnerton-Dyer conjecture for Hasse-Weil-Artin L-functions. J. Algebr. Geom. 24, 569-604 (2015)

12. Breuil, C., Conrad, B., Diamond, F., Taylor, R.: On the modularity of elliptic curves over $\mathbb{Q}$ : wild 3-adic exercises. J. Am. Math. Soc. 14(4), 843-939 (2001)

13. Darmon, H.: A refined conjecture of Mazur-Tate type for Heegner points. Invent. Math. 110, 123-146 (1992)

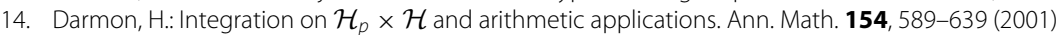

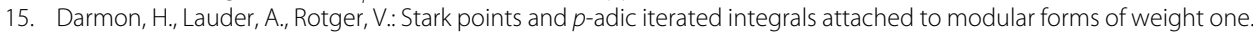
Forum Math. Pi 3, e8 (2015)

16. Darmon, H., Lauder, A., Rotger, V.: Gross-Stark units and $p$-adic iterated integrals attached to modular forms of weight one. Ann. Math. Qué. 40(2), 325-354 (2016)

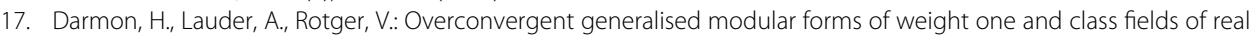
quadratic fields. Adv. Math. 283, 130-142 (2015)

18. Darmon, H., Rotger, V.: Diagonal cycles and Euler systems I: a p-adic Gross-Zagier formula. Ann. Sci. I'École Normale Supér. 47(4), 779-832 (2014)

19. Darmon, H., Rotger, V.: Diagonal cycles and Euler systems II: the Birch and Swinnerton-Dyer conjecture for Hasse-WeilArtin L-series. J. AMS (2016). doi:10.1090/jams/861

20. Darmon, H., Rotger, V.: Stark-Heegner points and generalised Kato classes, in progress

21. Gross, B., Zagier, D.: Heegner points and derivatives of L-series. Invent. Math. 84(2), 225-320 (1986)

22. Harris, M., Lan, K.-W., Taylor, R., Thorne, J.: On the rigid cohomology of certain Shimura varieties, preprint

23. Kato, K:: $p$-adic Hodge theory and values of zeta functions of modular forms. Cohomologies $p$-adiques et applications arithmétiques III Astérisque 295, ix (2004). (117-290) 
24. Kings, G., Loeffler, D., Zerbes, S.: Rankin-Selberg Euler systems and $p$-adic interpolation (submitted)

25. Kolyvagin, V.: Finiteness of $E(\mathbf{Q})$ and $\amalg(E, \mathbf{Q})$ for a subclass of Weil curves, Izv. Akad. Nauk SSSR Ser. Mat. $\mathbf{5 2}(3)$ 670-671 (1988); translation in Math. USSR-Izv. 32(3) 523-541 (1989)

26. Lauder, A.: Efficient computation of Rankin p-adic L-functions. In: Böckle, G. Wiese, G. (eds.) Computations with Modular Forms: Proceedings of a Summer School and Conference, Heidelberg, August/September 2011, pp. 181-200. Springer (2014)

27. Lei, A., Loeffler, D., Zerbes, S.L.: Euler systems for Rankin-Selberg convolutions of modular forms. Ann. Math. 180(2), 653-771 (2014)

28. Murty, M.R., Murty, V.K.: Non-vanishing of L-values and applications. In: Progress Math, vol. 157. Birkhäuser Verlag, Basel (1997)

29. Nekovár̆, J., Scholl, A.J.: Introduction to plectic cohomology. In: Jiang, D., Shahidi, F., Soudry, D. (eds.) Advances in the Theory of Automorphic Forms and Their L-functions. Contemporary Mathematics 664, pp. 321-337. American Mathematical Society, Providence, RI (2016)

30. Ohta, M.: On the $p$-adic Eichler-Shimura isomorphism for $\Lambda$-adic cusp forms. J. Reine Angew. Math. 463, 49-98 (1995)

31. Prasad, D.: Trilinear forms for representations of $\mathrm{GL}_{2}$ and local epsilon factors. Comput. Math. 75, 1-46 (1990)

32. Scholl, A.J.: An introduction to Kato's Euler systems. In: Galois Representations in Arithmetic Algebraic Geometry (Durham, 1996), London Mathematical Society Lecture Note Series 254, pp. 379-460. Cambridge University Press (1998)

33. Skinner, C.: A converse to a theorem of Gross, Zagier, and Kolyvagin, preprint, in arXiv:1405.7294

34. Skinner, C., Urban, E.: Vanishing of L-functions and ranks of Selmer groups. Int. Congr. Math. 2, 473-500 (2006)

35. Skinner, C., Urban, E.: The Iwasawa main conjectures for GL2. Invent. Math. 195(1), 1-277 (2014)

36. Stark, H.M.: L-functions at $s=1$. II. Artin L-functions with rational characters. Adv. Math. 17, 60-92 (1975)

37. Taylor, R., Wiles, A.: Ring-theoretic properties of certain Hecke algebras. Ann. Math. 141, 553-572 (1995)

38. Yuan, X., Zhang, S., Zhang, W.: Triple product $L$-series and Gross-Schoen cycles, preprint

39. Wan, X.: Iwasawa main conjecture for Rankin-Selberg p-adic L-functions, submitted, 2014. http://www.math. columbia.edu/ xw2295/paper2

40. Wiles, A.: Modular elliptic curves and Fermat's last theorem. Ann. Math. (2) 141(3), 443-551 (1995)

\section{Submit your manuscript to a SpringerOpen ${ }^{\circ}$ journal and benefit from:}

- Convenient online submission

- Rigorous peer review

- Immediate publication on acceptance

- Open access: articles freely available online

- High visibility within the field

- Retaining the copyright to your article

Submit your next manuscript at $\boldsymbol{s p r i n g e r o p e n . c o m ~}$ 